CQUeST-2010-0392

\title{
Mass and Angular momentum of Black Holes in New Massive Gravity
}

\author{
Soonkeon Nam¹, Jong-Dae Park?2, Sang-Heon Yi3 \\ Department of Physics and Research Institute of Basic Science, Kyung Hee University, \\ Seoul 130-701, Korea ${ }^{1,2}$ \\ Center for Quantum Spacetime, Sogang University, Seoul 121-741, Korea ${ }^{3}$
}

\begin{abstract}
We obtain mass and angular momentum of black holes as conserved charges in three dimensional new massive gravity, after presenting the explicit expression for the potential of the conserved charges. This confirms the expression of those charges obtained in several ways, in particular through AdS/CFT correspondence, and shows us that the first law of black hole thermodynamics is valid in these black holes. We also comment about conserved charges in new type black holes with the emphasis on the AdS/CFT correspondence as guiding principle.
\end{abstract}

\footnotetext{
${ }^{1}$ nam@khu.ac.kr

2jdpark@khu.ac.kr

${ }^{3}$ shyi@sogang.ac.kr
} 


\section{Introduction}

Three dimensional pure Einstein gravity is non-dynamical and seems not so relevant to our dynamical four dimensional gravity. Despite of this apparent irrelevance, three dimensional gravity becomes an interesting arena toward understanding some aspects of gravity and testing various ideas about black holes, since it may contain black holes and have a dual description through $A d S / C F T$ correspondence. Furthermore, when additional terms such as higher curvature terms are added, its physical contents become richer and it has some interesting aspects in the viewpoint of $A d S / C F T$ correspondence. The higher curvature terms in three dimension make gravitons propagate and open a new window of the $A d S / C F T$ correspondence.

Gravitational Chern-Simons term is one of interesting correction terms, for which it has long been known that three dimensional pure gravity with this term [1] 2] leads to massive gravitons, allows black hole solutions, and also has a string theory embedding [3] [10]. This three dimensional gravity with Chern-Simons term has been called topologically massive gravity(TMG). This TMG has also been investigated in the viewpoint of $A d S / C F T$ correspondence(See [11] 14]). Recently, another type of three dimensional massive gravity theory with a specific combination of higher curvature terms has been explored (see for a review [15] [19]), which is called new massive gravity(NMG). The simplest version of this theory contains a specific combination of scalar curvature square and Ricci tensor square, and it preserves parity symmetry contrary to TMG. It has also been shown that this NMG allows various black holes [16] [25] and also has another realization of the $A d S / C F T$ correspondence.

Though there are several studies on black holes in NMG, far less is known than those in TMG. Mass and angular momentum of black holes are not obtained as conserved charges and the so-called new type black holes found in NMG are not so well understood, yet. In this paper, we obatin mass and angular momentum as conserved charges on various black holes and then resolve some issues on new type black holes.

There are several methods to define mass and angular momentum of black holes for higher curvature theories, for example as in [26] 33]. One of the easiest way to obtain those in the case of asymptotically $A d S$ space is to resort to AdS/CFT correspondence. Specifically, in the three dimensional case the mass and angular momentum of asymptotically $A d S$ black holes can be obtained by

$$
M=E_{L}+E_{R}, \quad J=L\left(E_{L}-E_{R}\right),
$$

where $E_{L}, E_{R}$ are left and right energies of the dual CFT and $L$ is the $A d S$ radius. In terms of the central charges of dual two dimensional CFTs, these energies can be related to the left and right black hole temperatures as $E_{L}=\pi^{2} L / 6 \cdot c_{L} T_{L}^{2}, E_{R}=\pi^{2} L / 6 \cdot c_{R} T_{R}^{2}$, and central charges of the conjectural dual CFTs can also be obtained from black hole solutions through the Cardy formula. Therefore, the above formulae mean that mass and angular momentum can be obtained purely from gravity data without CFT. For warped $A d S$ black holes, those should be read as

$$
M=\sqrt{\frac{3 \beta^{4}}{4 \beta^{2}-1}} \sqrt{\frac{2 c_{L} E_{L}}{3 L}}, \quad J=L\left(E_{L}-E_{R}\right),
$$

where $\beta$ is the warp factor. 
These formulae for obtaining mass and angular momentum through $A d S / C F T$ correspondence are consistent with the first law of black holes thermodynamics by construction. These formulae also hold in TMG for the same kind black holes, and can be shown to be identical with those obtained as conserved charges. One of our main results is to verify these formulae in NMG by obtaining mass and angular momentum of black holes as conserved charges in the purely gravity context, which can also be understood as verification of the first law of black hole thermodynamics for these black holes in NMG.

In NMG there are new black hole solutions, so-called new type black holes, in addition to the above mentioned (warped) AdS black holes [16]. Though these solutions are obtained analytically, a deep understanding of their physical properties is still lacking. One of motivations to study conserved charges of black holes in this paper is to improve this situation in these black holes, keeping in mind $A d S / C F T$ correspondence as the guiding principle.

The asymptotic of new type black holes in NMG is $A d S$ space, which is identical to the asymptotic of BTZ black holes. Therefore, according to the standard AdS/CFT correspondence, new type black holes and BTZ black holes should correspnd to the related dual CFTs, while the dual CFTs need not be completely identical. In particular, the central charges obtained from new type black holes and BTZ black holes should be matched for a suitable adjustment of parameters which is necessary for the existence of new type black holes in the gravity Lagrangian. This matching of the central charges was confirmed as that is the case in Ref. [33]. It seems that this correspondence allows one to obtain conserved charges for new type black holes through the formulae for the BTZ case Eq. (11). However, mass of new type black holes obtained as conserved charge in [24] is not consistent with this argument. We will resolve this issue by reexamining mass of new type black holes as conserved charge by comparing the procedure in the warped $A d S$ black hole case.

The following sections are organized as follows. In section 2, we briefly review the so-called ADT currents, potential and charges [26] [27] [28], and present their explicit expressions for NMG. We give the generic expression of the ADT potential for scalar curvature square and Ricci tensor square, which is applicable in any dimension. We obtain the vector expression for ADT charges, in $S L(2, \mathbf{R})$ reduction formalism, of (warped) $A d S$ black holes and its relation with super angular momentum in section 3. In this section, we present mass and angular momentum of black holes as conserved charges. This confirms the suggested expression for these charges and verifies the first law of black hole thermodynamics. In section 4, we comment on conserved charges for new type black holes. In the final section, we summarize our results with some comments on our approach and on the open issues. Some calculational details are given in the Appendices.

\section{Conserved Charges in New Massive Gravity}

Usually, it is not so straightforward to define conserved charges in a theory with general covariance, since the concept of conservation requires the choice of time coordinate but general covariance denies the preferred time coordinate. However, for asymptotically fixed spacetime, it seems to be manageable to define time coordinate in a canonical way. For instance, asymptotically flat spacetime allows so-called ADM formalism. In the case of asymptotically $A d S$ spacetime, one needs 
to devise another approach. In the following subsection, we will review the approach pioneered by Abbott, Deser and Tekin (ADT) [26] 27] [28, and successfully applied to the TMG case [6] 34]. In the next subsection, we present our results of ADT charges in the NMG case.

\subsection{Review: ADT formalism}

One way to define conserved charges in gravity theory is to construct covariantly conserved currents and to integrate these currents. In this subsection, we review the so-called ADT currents, ADT charges and their construction in terms of the linearized Bianchi identity and a Killing vector. This process with minimum assumption in TMG is given in [6], which is adopted and presented in our convention.

An arbitrary metric, $g_{\mu \nu}$ can be expanded around the background metric $\bar{g}_{\mu \nu}$ as

$$
g_{\mu \nu}=\bar{g}_{\mu \nu}+h_{\mu \nu}
$$

where the background metric will be taken as the solution of equations of motion (EOM)

$$
\mathcal{E}_{\mu \nu}(\bar{g})=0 .
$$

In the following, we will call the left hand side expression of the above equation, $\mathcal{E}_{\mu \nu}$, as the EOM expression. Note that the EOM expression need not vanish for a generic metric $g_{\mu \nu}$.

For any kind of gravitational theory without matter, the general covariance of gravity leads to the following differential Bianchi identity for an arbitrary metric $g_{\mu \nu}$ as

$$
\nabla_{\mu} \mathcal{E}^{\mu \nu}=0
$$

which imposes the linearized Bianchi identity on the linearized EOM expression, $\delta \mathcal{E}^{\mu \nu}$, through EOM for the background metric $\bar{g}_{\mu \nu}$, as

$$
\bar{\nabla}_{\mu} \delta \mathcal{E}^{\mu \nu}=0
$$

Note that this expression is nothing but the equation for the covariant conservation except that the conserved quantity is a symmetric tensor, not a vector. From now on, the bar notation is dropped and so the covariant derivatives and curvatures are referred to the background metric for our convenience.

The ADT currents are defined by the contraction of the linearized EOM expression, $\delta \mathcal{E}$ and a Killing vector $\xi$ as

$$
J^{\mu} \equiv \delta \mathcal{E}^{\mu \nu} \xi_{\nu}
$$

One can see that these currents are conserved covariantly by the linearized Bianchi identity of $\delta \mathcal{E}^{\mu \nu}$ and the Killing property of $\xi_{\nu}$. Under EOM of the background metric, antisymmetric tensor potential, $Q^{\mu \nu}$ for this current can defined by

$$
J^{\mu}=\nabla_{\nu} Q^{\mu \nu}
$$


which guarantees current conservation by the antisymmetric property of the potential $Q^{\mu \nu}$. Though this is a legitimate way to obtain the potential of currents, it is not convenient one, since we should impose EOM to define the potential.

The current, itself, may be defined without EOM although its conservation requires EOM, and it is not so favorable to use EOM for a generic higher curvature theory. Therefore, it is desirable to define the potential, $Q$ without EOM. That is to say, the potential needs to be defined for a generic background. This can be achieved by defining the antisymmetric tensor potential, $Q^{\mu \nu}$ for the current as [6]

$$
J^{\mu}=\delta \mathcal{E}^{\mu \nu} \xi_{\nu} \equiv-\mathcal{E}^{\mu \alpha} h_{\alpha \nu} \xi^{\nu}+\frac{1}{2} \xi^{\mu} \mathcal{E}^{\alpha \beta} h_{\alpha \beta}-\frac{1}{2} h \mathcal{E}_{\nu}^{\mu} \xi^{\nu}+\nabla_{\nu} Q^{\mu \nu}
$$

which reduces the previous definition of the potential when EOM is imposed.

Noting

$$
\delta \mathcal{E}^{\mu \nu} \xi_{\nu}=-h^{\mu \alpha} \mathcal{E}_{\alpha \nu} \xi^{\nu}-\mathcal{E}^{\mu \alpha} h_{\alpha \nu} \xi^{\nu}+g^{\mu \alpha} \delta \mathcal{E}_{\alpha \nu} \xi^{\nu}
$$

one can see that

$$
g^{\mu \alpha} \delta \mathcal{E}_{\alpha \nu} \xi^{\nu}=h^{\mu \alpha} \mathcal{E}_{\alpha \nu} \xi^{\nu}+\frac{1}{2} \xi^{\mu} \mathcal{E}^{\alpha \beta} h_{\alpha \beta}-\frac{1}{2} h \mathcal{E}_{\nu}^{\mu} \xi^{\nu}+\nabla_{\nu} Q^{\mu \nu}
$$

This is the form that we will utilize to obtain the ADT potential and subsequently ADT charges.

In terms of this potential for the current, ADT charges for a Killing vector $\xi$ is given by

$$
Q(\xi)=\frac{1}{2 \kappa^{2}} \int d \Sigma_{\mu \nu} Q^{\mu \nu}(\xi)
$$

where $2 \kappa^{2}=16 \pi G$ is the Newton's constant.

Using the ADT potential and denoting time, radial and angle coordinates as $(t, r, \phi)$, one obtains mass and angular momentum in the three dimensional case of our interest as

$$
M=\left.\frac{1}{4 G} \sqrt{-\operatorname{det} g} Q^{r t}\left(\xi_{T}\right)\right|_{r \rightarrow \infty}, \quad J=\left.\frac{1}{4 G} \sqrt{-\operatorname{det} g} Q^{r t}\left(\xi_{R}\right)\right|_{r \rightarrow \infty},
$$

where $\xi_{T}$ and $\xi_{R}$ denote the time translational and rotational Killing vector, respectively. Note that the normalization of Killing vectors has effects on the overall scale of conserved charges. So, we will adopt the convention used in 33 such that in the case of asymptotic AdS space of the radius $L$ normalized at the spacelike infinity as $\xi_{T}^{2} \rightarrow-r^{2}$ and $\xi_{R}^{2} \rightarrow L^{2} r^{2}$ with $g_{t t} \rightarrow-L^{2} r^{2}$ and $g_{\phi \phi} \rightarrow L^{2} r^{2}$, Killing vectors are taken as

$$
\xi_{T}=\frac{1}{L} \frac{\partial}{\partial t}, \quad \xi_{R}=\frac{\partial}{\partial \phi},
$$

which is the convention we will use in the following.

We will illustrate the above procedure for the Einstein-Hilbert term with cosmological constant in the Lagrangian, $\mathcal{L}=R+\frac{2}{l^{2}}$. The metric variation leads to EOM expression as

$$
\mathcal{E}_{\mu \nu}=R_{\mu \nu}-\frac{1}{2} g_{\mu \nu}\left(R+\frac{2}{l^{2}}\right) .
$$


To obtain the ADT potential for ADT currents, we compute the linearized EOM expression with the contraction of a Killing vector $\xi$ in Eq. (8) as

$$
g^{\mu \rho} \xi^{\nu} \delta \mathcal{E}_{\rho \nu}^{R}=-\frac{1}{2} h_{\nu}^{\mu} \xi^{\nu}\left(R+\frac{2}{l^{2}}\right)+g^{\mu \rho} \xi^{\nu}\left(\delta R_{\rho \nu}-\frac{1}{2} g_{\mu \nu} \delta R\right) .
$$

For our convenience, let us introduce

$$
\mathcal{I}_{\mu \nu} \equiv \delta R_{\mu \nu}-\frac{1}{2} g_{\mu \nu} \delta R
$$

which satisfies

$$
\begin{aligned}
\mathcal{I}_{\nu}^{\mu} \xi^{\nu} & =g^{\mu \alpha}\left(\delta R_{\alpha \beta}-\frac{1}{2} g_{\alpha \beta} \delta R\right) \xi^{\beta} \\
& =\frac{1}{2} \xi^{\nu}\left(\nabla^{\alpha} \nabla^{\mu} h_{\nu \alpha}+\nabla^{\alpha} \nabla_{\nu} h_{\alpha}^{\mu}-\nabla^{2} h_{\nu}^{\mu}-\nabla^{\mu} \nabla_{\nu} h\right)-\frac{1}{2} \xi^{\mu}\left(\nabla^{\alpha} \nabla^{\beta} h_{\alpha \beta}-\nabla^{2} h-h_{\alpha \beta} R^{\alpha \beta}\right) .
\end{aligned}
$$

After combining terms to form antisymmetric total derivatives through Leibniz's rule, one obtains

$$
\mathcal{I}^{\mu} \xi^{\nu}=\nabla_{\nu} Q_{R}^{\mu \nu}+\frac{1}{2} \xi^{\mu} h_{\alpha \beta} R^{\alpha \beta}+\frac{1}{2} h \nabla^{2} \xi^{\mu}+\frac{1}{2} \xi^{\nu}\left[\nabla^{\alpha}, \nabla_{\nu}\right] h_{\alpha}^{\mu}+\frac{1}{2} h^{\nu \alpha} \nabla_{\nu} \nabla^{\mu} \xi_{\alpha}-\frac{1}{2} h^{\mu \alpha} \nabla^{2} \xi_{\alpha},
$$

where

$$
Q_{R}^{\mu \nu} \equiv \xi_{\alpha} \nabla^{[\mu} h^{\nu] \alpha}-\xi^{[\mu} \nabla_{\alpha} h^{\nu] \alpha}-h^{\alpha[\mu} \nabla_{\alpha} \xi^{\nu]}+\xi^{[\mu} \nabla^{\nu]} h+\frac{1}{2} h \nabla^{[\mu} \xi^{\nu]} .
$$

Using the properties of Killing vectors and the definition of Riemann tensors, one obtains

$$
\mathcal{I}^{\mu} \xi^{\nu}=\nabla_{\nu} Q_{R}^{\mu \nu}+h^{\mu \alpha} R_{\alpha \nu} \xi^{\nu}+\frac{1}{2} \xi^{\mu} R^{\alpha \beta} h_{\alpha \beta}-\frac{1}{2} R_{\nu}^{\mu} \xi^{\nu} h
$$

As a result, one can see that

$$
g^{\mu \rho} \xi^{\nu} \delta \mathcal{E}_{\rho \nu}^{R}=h^{\mu \alpha} \mathcal{E}_{\alpha \nu}^{R} \xi^{\nu}+\frac{1}{2} \xi^{\mu} \mathcal{E}_{R}^{\alpha \beta} h_{\alpha \beta}-\frac{1}{2} h \mathcal{E}_{R \nu}^{\mu} \xi^{\nu}+\nabla_{\nu} Q_{R}^{\mu \nu},
$$

and that the ADT potential for Einstein-Hilbert terms is given by the above $Q_{R}^{\mu \nu}$.

\subsection{ADT Potential in NMG}

Now, let us consider ADT potentials in NMG. We will consider the simplest version of NMG of which action is given by 19 ]

$$
S=\frac{\eta}{2 \kappa^{2}} \int d^{3} x \sqrt{-g}\left[\sigma R+\frac{2}{l^{2}}+\frac{1}{m^{2}} K\right],
$$

where $\eta$ and $\sigma$ take 1 or -1 , and $K$ is defined by

$$
K=R_{\mu \nu} R^{\mu \nu}-\frac{3}{8} R^{2}
$$

\footnotetext{
${ }^{1}$ We have introduced $\eta$ for the various sign choice of terms in the action.
} 
Our convention is such that $m^{2}$ is always positive but the cosmological constant $l^{2}$ has no such restriction. The EOM of NMG is given by

$$
\mathcal{E}_{\mu \nu}=\eta\left[\sigma G_{\mu \nu}-\frac{1}{l^{2}} g_{\mu \nu}+\frac{1}{2 m^{2}} K_{\mu \nu}\right]=0,
$$

where

$$
K_{\mu \nu}=g_{\mu \nu}\left(3 R_{\alpha \beta} R^{\alpha \beta}-\frac{13}{8} R^{2}\right)+\frac{9}{2} R R_{\mu \nu}-8 R_{\mu \alpha} R_{\nu}^{\alpha}+\frac{1}{2}\left(4 \nabla^{2} R_{\mu \nu}-\nabla_{\mu} \nabla_{\nu} R-g_{\mu \nu} \nabla^{2} R\right) .
$$

Since the ADT potential is additive, it is sufficient to present the ADT potential for $K$-term. The ADT potential for the $K$-term may also be obtained by adding the contribution from $R^{2}$ term and $R_{2} \equiv R_{\mu \nu} R^{\mu \nu}$ term. In the following we present the ADT potential for $R^{2}$ and $R_{2}$, respectively. At this stage our results are independent of dimension. Now, let us consider each contribution separately. For the $R^{2}$ term, the EOM expression is given by

$$
\mathcal{E}_{\mu \nu}^{R^{2}}=2\left[R R_{\mu \nu}+g_{\mu \nu} \nabla^{2} R-\nabla_{\mu} \nabla_{\nu} R\right]-\frac{1}{2} g_{\mu \nu} R^{2} .
$$

It is straightforward to obtain its variation as

$$
\begin{aligned}
\frac{1}{2} \delta \mathcal{E}_{\mu \nu}^{R^{2}=} & h_{\mu \nu}\left(\nabla^{2} R-\frac{1}{4} R^{2}\right)+\delta R R_{\mu \nu}+g_{\mu \nu} \nabla^{2} \delta R-\nabla_{\mu} \nabla_{\nu} \delta R+R \mathcal{I}_{\mu \nu} \\
& +g_{\mu \nu}\left[-h_{\alpha \beta} \nabla^{\alpha} \nabla^{\beta} R-g^{\alpha \beta} \delta \Gamma_{\alpha \beta}^{\nu} \nabla_{\nu} R\right]+\delta \Gamma_{\mu \nu}^{\alpha} \nabla_{\alpha} R .
\end{aligned}
$$

Noting that

$$
\xi^{\mu} \nabla^{2} \delta R-\xi^{\nu} \nabla^{\mu} \nabla_{\nu} \delta R=\nabla_{\nu}\left[2 \xi^{[\mu} \nabla^{\nu]} \delta R+\delta R \nabla^{[\mu} \xi^{\nu]}\right]-\delta R R_{\nu}^{\mu} \xi^{\nu},
$$

one can see that

$$
\begin{aligned}
\frac{1}{2} g^{\mu \alpha} \delta \mathcal{E}_{\alpha \beta}^{R^{2}} \xi^{\beta}= & \nabla_{\nu}\left[2 \xi^{[\mu} \nabla^{\nu]} \delta R+\delta R \nabla^{[\mu} \xi^{\nu]}\right]+h_{\nu}^{\mu} \xi^{\nu}\left(\nabla^{2} R-\frac{1}{4} R^{2}\right)+R \mathcal{I}_{\nu}^{\mu} \xi^{\nu} \\
& -\xi^{\mu}\left[h_{\alpha \beta} \nabla^{\alpha} \nabla^{\beta} R+g^{\alpha \beta} \delta \Gamma_{\alpha \beta}^{\nu} \nabla_{\nu} R\right]+g^{\mu \rho} \xi^{\nu} \delta \Gamma_{\rho \nu}^{\alpha} \nabla_{\alpha} R,
\end{aligned}
$$

Using the following formula

$$
\begin{aligned}
& -\xi^{\mu}\left[h_{\alpha \beta} \nabla^{\alpha} \nabla^{\beta} R+g^{\alpha \beta} \delta \Gamma_{\alpha \beta}^{\nu} \nabla_{\nu} R\right]+g^{\mu \rho} \xi^{\nu} \delta \Gamma_{\rho \nu}^{\alpha} \nabla_{\alpha} R \\
& =-\nabla_{\nu}\left[\xi^{[\mu} h^{\nu] \alpha} \nabla_{\alpha} R\right]+Q_{R}^{\mu \nu} \nabla_{\nu} R-h^{\mu \alpha} \xi^{\nu} \nabla_{\alpha} \nabla_{\nu} R+\frac{1}{2} h \xi^{\nu} \nabla^{\mu} \nabla_{\nu} R-\frac{1}{2} \xi^{\mu} h^{\alpha \beta} \nabla_{\alpha} \nabla_{\beta} R,
\end{aligned}
$$

and recalling the identity (14) with Leibniz's rule, one can see that the ADT potential for the contribution of $R^{2}$ term is given by

$$
Q_{R^{2}}^{\mu \nu}=2 R Q_{R}^{\mu \nu}+4 \xi^{[\mu} \nabla^{\nu]} \delta R+2 \delta R \nabla^{[\mu} \xi^{\nu]}-2 \xi^{[\mu} h^{\nu] \alpha} \nabla_{\alpha} R .
$$

where $Q_{R}^{\mu \nu}$ is defined in Eq. (13) and $\delta R$ means that

$$
\delta R \equiv-R^{\alpha \beta} h_{\alpha \beta}+\nabla^{\alpha} \nabla^{\beta} h_{\alpha \beta}-\nabla^{2} h .
$$


Now, let us turn to the contribution of $R_{2} \equiv R_{\mu \nu} R^{\mu \nu}$ term to the ADT potential in $K$-term. The EOM expression of $R_{2}$ term is given by

$$
\mathcal{E}_{\mu \nu}^{R_{2}}=-2 R_{\mu \alpha \beta \nu} R^{\alpha \beta}+\nabla^{2} R_{\mu \nu}-\nabla_{\mu} \nabla_{\nu} R+\frac{1}{2} g_{\mu \nu}\left(\nabla^{2} R-R_{\alpha \beta} R^{\alpha \beta}\right),
$$

and its variation is

$$
\begin{aligned}
\delta \mathcal{E}_{\mu \nu}^{R_{2}}= & \frac{1}{2} h_{\mu \nu}\left(\nabla^{2} R-R_{\alpha \beta} R^{\alpha \beta}\right)-2\left(\delta R_{\mu \alpha \beta \nu} R^{\alpha \beta}+R_{\mu \alpha \beta \nu} \delta R^{\alpha \beta}\right)-\frac{1}{2} g_{\mu \nu} \delta\left(R_{\alpha \beta} R^{\alpha \beta}\right) \\
& +g_{\mu \nu} \nabla^{2} \delta R-\nabla_{\mu} \nabla_{\nu} \delta R+\nabla^{2} \mathcal{I}_{\mu \nu}-\nabla^{\alpha}\left(h_{\alpha \beta} \nabla^{\beta} R_{\mu \nu}\right)+\frac{1}{2} \nabla_{\alpha} h \nabla^{\alpha} R_{\mu \nu}+F_{\mu \nu} \\
& -2 \delta \Gamma_{\alpha \mu}^{\beta} \nabla^{\alpha} R_{\beta \nu}-2 \delta \Gamma_{\alpha \nu}^{\beta} \nabla^{\alpha} R_{\beta \mu}+\frac{1}{2} g_{\mu \nu}\left[-\nabla^{\alpha}\left(h_{\alpha \beta} \nabla^{\beta} R\right)+\frac{1}{2} \nabla_{\alpha} h \nabla^{\alpha} R\right]+\delta \Gamma_{\mu \nu}^{\alpha} \nabla_{\alpha} R,
\end{aligned}
$$

where

$$
F_{\mu \nu} \equiv-\nabla^{\alpha} \delta \Gamma_{\alpha \mu}^{\beta} R_{\beta \nu}-\nabla^{\alpha} \delta \Gamma_{\alpha \nu}^{\beta} R_{\beta \mu}
$$

To obtain ADT potential according to (8) in the squared Ricci curvature case, let us write the contraction of linearized EOM expression with a Killing vector as

$$
g^{\mu \rho} \delta \mathcal{E}_{\rho \nu} \xi^{\nu}=\frac{1}{2} h_{\nu}^{\mu} \xi^{\nu}\left(\nabla^{2} R-R_{\alpha \beta} R^{\alpha \beta}\right)+E_{1}^{\mu}+E_{2}^{\mu}+E_{3}^{\mu}+E_{4}^{\mu}+E_{5}^{\mu},
$$

where

$$
\begin{aligned}
E_{1}^{\mu} & \equiv-2 g^{\mu \rho} \xi^{\nu} \delta\left(R_{\rho \alpha \beta \nu} R^{\alpha \beta}\right)-\frac{1}{2} \xi^{\mu} \delta\left(R_{\alpha \beta} R^{\alpha \beta}\right)-2 \xi^{\nu} \delta \Gamma_{\alpha \nu}^{\beta} \nabla^{\alpha} R_{\beta}^{\mu}, \\
E_{2}^{\mu} & \equiv \xi^{\mu} \nabla^{2} \delta R-\xi^{\nu} \nabla_{\nu} \nabla^{\mu} \delta R+\xi^{\nu} \nabla^{2} \mathcal{I}_{\nu}^{\mu}-g^{\mu \rho} \xi^{\nu} R_{\nu \beta} \nabla^{\alpha} \delta \Gamma_{\alpha \rho}^{\beta}-\xi^{\nu} R_{\beta}^{\mu} \nabla^{\alpha} \delta \Gamma_{\alpha \nu}^{\beta}, \\
E_{3}^{\mu} & \equiv-2 g^{\mu \rho} \xi^{\nu} \delta \Gamma_{\alpha \rho}^{\beta} \nabla^{\alpha} R_{\beta \nu}-\xi^{\nu} \nabla_{\alpha}\left(h^{\alpha \beta} \nabla_{\beta} R_{\nu}^{\mu}\right), \\
E_{4}^{\mu} & \equiv-\frac{1}{2} \xi^{\mu} \nabla_{\alpha}\left(h^{\alpha \beta} \nabla_{\beta} R\right)+g^{\mu \rho} \xi^{\nu} \delta \Gamma_{\rho \nu}^{\alpha} \nabla_{\alpha} R, \\
E_{5}^{\mu} & \equiv \frac{1}{2} \xi^{\nu} \nabla_{\alpha} h \nabla^{\alpha} R_{\nu}^{\mu}+\frac{1}{4} \xi^{\mu} \nabla_{\alpha} h \nabla^{\alpha} R .
\end{aligned}
$$

Collecting various contributions from each $E_{a}^{\mu}$ term which can be obtained by using formulae in the Appendix A, one can obtain the contribution of the squared Ricci curvature $R_{2}=R_{\mu \nu} R^{\mu \nu}$ to the ADT potential as

$$
\begin{aligned}
Q_{R_{2}}^{\mu \nu}= & 2 \xi^{\alpha} R^{\beta[\mu} \nabla_{\beta} h_{\alpha}^{\nu]}-2 \xi^{\alpha} R^{\beta[\mu} \nabla^{\nu]} h_{\alpha \beta}+2 R^{\alpha \beta} \xi^{[\mu} \nabla^{\nu]} h_{\alpha \beta}-2 R^{\alpha \beta} \xi^{[\mu} \nabla_{\alpha} h_{\beta}^{\nu]}+2 h^{\alpha \beta} \nabla_{\alpha}\left(\xi^{[\mu} R_{\beta}^{\nu]}\right) \\
& +R^{\mu \nu}{ }_{\alpha \beta} \xi^{\alpha} \nabla_{\gamma} h^{\gamma \beta}-2 \xi^{\gamma} R_{\gamma}^{\alpha \beta[\mu} \nabla_{\alpha} h_{\beta}^{\nu]}+2 \xi^{\gamma} R_{\gamma}^{\alpha \beta[\mu} \nabla^{\nu]} h_{\alpha \beta} \\
& +\nabla^{2} Q_{R}^{\mu \nu}+R_{\alpha \beta}^{\mu \nu} Q_{R}^{\alpha \beta}+2 Q_{R}^{\alpha[\mu} R_{\alpha}^{\nu]}+2 \xi^{[\mu} \nabla^{\nu]} \delta R-2 \xi^{\alpha} R_{\alpha}^{[\mu} \nabla_{\beta} h^{\nu] \beta}-2 \nabla^{\alpha} \xi^{\beta} \nabla_{\alpha} \nabla^{[\mu} h_{\beta}^{\nu]} \\
& +2 \xi^{\beta} h^{\alpha[\mu} \nabla_{\alpha} R_{\beta}^{\nu]}-\xi^{[\mu} h^{\nu] \alpha} \nabla_{\alpha} R \\
& +2 R_{\alpha \beta} h^{\alpha[\mu} \nabla^{\nu]} \xi^{\beta}-2 h_{\alpha \beta} R_{\gamma}^{\alpha \beta[\mu} \nabla^{\nu]} \xi^{\gamma} \\
& -R^{\mu \nu}{ }_{\alpha \beta} \xi^{[\alpha} \nabla^{\beta]} h+\xi^{\alpha} R_{\alpha}^{[\mu} \nabla^{\nu]} h+\xi^{[\mu} R^{\nu] \alpha} \nabla_{\alpha} h-2 h R_{\alpha}^{[\mu} \nabla^{\nu]} \xi^{\alpha} .
\end{aligned}
$$

Using three dimensional identity, $R_{\alpha \beta}^{\mu \nu}=2\left(\delta_{\alpha}^{[\mu} R_{\beta}^{\nu]}-\delta_{\beta}^{[\mu} R_{\alpha}^{\nu]}\right)-R \delta_{\alpha}^{[\mu} \delta_{\beta}^{\nu]}$, one may rewrite $Q_{R_{2}}^{\mu \nu}$ as

$$
\begin{aligned}
Q_{R_{2}}^{\mu \nu}= & \nabla^{2} Q_{R}^{\mu \nu}+\frac{1}{2} Q_{R^{2}}^{\mu \nu}-2 Q_{R}^{\alpha[\mu} R_{\alpha}^{\nu]}-2 \nabla^{\alpha} \xi^{\beta} \nabla_{\alpha} \nabla^{[\mu} h_{\beta}^{\nu]}-4 \xi^{\alpha} R_{\alpha \beta} \nabla^{[\mu} h^{\nu] \beta}-R h_{\alpha}^{[\mu} \nabla^{\nu]} \xi^{\alpha} \\
& +2 \xi^{[\mu} R_{\alpha}^{\nu]} \nabla_{\beta} h^{\alpha \beta}+2 \xi_{\alpha} R^{\alpha[\mu} \nabla_{\beta} h^{\nu] \beta}+2 \xi^{\alpha} h^{\beta[\mu} \nabla_{\beta} R_{\alpha}^{\nu]}+2 h^{\alpha \beta} \xi^{[\mu} \nabla_{\alpha} R_{\beta}^{\nu]} \\
& -\left(\delta R+2 R^{\alpha \beta} h_{\alpha \beta}\right) \nabla^{[\mu} \xi^{\nu]}-3 \xi^{\alpha} R_{\alpha}^{[\mu} \nabla^{\nu]} h-\xi^{[\mu} R^{\nu] \alpha} \nabla_{\alpha} h .
\end{aligned}
$$


One of our main results is the explicit expression for the ADT potential for the $K$-term in NMG, which is given by the sum of two expressions in Eq. (22) and Eq. (28) as

$$
Q_{K}^{\mu \nu}=Q_{R_{2}}^{\mu \nu}-\frac{3}{8} Q_{R^{2}}^{\mu \nu}
$$

In the following sections, we give the $\mathrm{ADT}$ potential $Q_{R}^{\mu \nu}$ and $Q_{K}^{\mu \nu}$ for each Killing vector, $\xi_{T}$ and $\xi_{R}$ on various black holes. The mass and angular momentum obtained by these potentials confirm suggested expression for those quantities.

\section{Mass and Angular Momentum as Conserved Charges}

In this section we will relate ADT charges or potential to super angular momentum by Clement. Before presenting our results, we review for completeness briefly $S L(2, \mathbf{R})$ reduced action method which is useful particularly for the three dimensional gravity [20] 35] 36] After that, we present the so-called correction term to mass in super angular momentum formalism by using the explicit expression of ADT potential.

\section{1 $S L(2, \mathbf{R})$ Reduction Method}

Let us take the three dimensional metric ansatz as

$$
d s^{2}=\lambda_{a b}(\rho) d x^{a} d x^{b}+\frac{d \rho^{2}}{\zeta^{2} U^{2}(\rho)}, \quad x^{a}=(t, \phi) .
$$

where all variables are functions only of the radial coordinate $\rho$. By the reparametrization invariance with respect to $\rho$ coordinate, function $U$ may be chosen such that the condition $\operatorname{det} \lambda=-U^{2}$ is satisfied. This choice implies that $\sqrt{-g}=1 / \zeta$. This metric ansatz reduces the given generally covariant Lagrangian to the $S L(2, \mathbf{R}) \simeq S O(1,2)$ invariant one. This means that the relevant variables becomes the $S O(1,2)$ three dimensional vectors and differential geometric calculation reduces to the vector calculus. In particular, Einstein equations reduces to the equations obtained by the variation with respect to the two dimensional metric $\lambda$ and the one with respect to $\zeta$. These equations are called EOM and Hamiltonian constraint, respectively in this $S L(2, \mathbf{R})$ reduction formalism. Using the vector expression for EOM, one may integrate EOM to obtain some conserved quantities, which is the analog of angular momentum in mechanical problem: This quantity is named as super angular momentum [3].

Explicitly, one may parameterize generic symmetric two by two matrix $\lambda$ as

$$
\lambda_{a b}=\left(\begin{array}{cc}
X^{0}+X^{1} & X^{2} \\
X^{2} & X^{0}-X^{1}
\end{array}\right),
$$

and associate a $S O(1,2)$ three dimensional vector $\mathbf{X}=\left(X^{0}, X^{1}, X^{2}\right)$ with this matrix. Conversely, the associated matrix with a vector $\mathbf{X}$ is denoted by $\langle\mathbf{X}\rangle$ and given explicitly by

$$
\langle\mathbf{X}\rangle=\boldsymbol{\tau} \cdot \mathbf{X}=\left(\begin{array}{cc}
-X^{2} & -X^{0}+X^{1} \\
X^{0}+X^{1} & X^{2}
\end{array}\right),
$$


where

$$
\tau^{0}=\left(\begin{array}{rr}
0 & -1 \\
1 & 0
\end{array}\right), \quad \tau^{1}=\left(\begin{array}{ll}
0 & 1 \\
1 & 0
\end{array}\right), \quad \tau^{2}=\left(\begin{array}{rr}
-1 & 0 \\
0 & 1
\end{array}\right) .
$$

Note that $\mathbf{X}^{2}=-\operatorname{det} \lambda=U^{2}$. Then, the above matrix $\lambda$ can be written as $\lambda=\tau^{0}\langle\mathbf{X}\rangle$ and its inverse is given by $\lambda^{-1}=-U^{-2}\langle\mathbf{X}\rangle \tau^{0}$. In terms of $\lambda$ and $U$, the connections of the above metric ansatz is given by

$$
\left.\Gamma_{a b}^{\rho}=-\frac{\zeta^{2}}{2} U^{2} \lambda_{a b}^{\prime}, \quad \Gamma_{\rho a}^{b}=\frac{1}{2}\left(\lambda^{-1} \lambda^{\prime}\right)_{a}^{b}, \quad \Gamma_{\rho \rho}^{\rho}=-\frac{U^{\prime}}{U}, \quad \quad{ }^{\prime} \equiv \frac{d}{d \rho}\right)
$$

which are subsequently written in terms of $\mathbf{X}$ and its derivatives. All other relevant differential geometric quantities such as the deviation metric $h$ and curvatures can be written in terms of vector $\mathbf{X}$ and its derivatives. For this purpose, it is useful to define the inner and cross product of $S O(1,2)$ vectors in the standard way as

$$
\mathbf{A} \cdot \mathbf{B}=\eta_{i j} A^{i} B^{j}, \quad(\mathbf{A} \times \mathbf{B})^{i}=\eta^{i m} \epsilon_{m j k} A^{j} B^{k}, \quad\left(\epsilon_{012}=1\right)
$$

and note that the product of two matrices dual to vectors $\mathbf{A}$ and $\mathbf{B}$ is given by

$$
\langle\mathbf{A}\rangle\langle\mathbf{B}\rangle=(\mathbf{A} \cdot \mathbf{B}) \mathbf{1}+\langle\mathbf{A} \times \mathbf{B}\rangle .
$$

For instance, one can see that2,

$$
\begin{aligned}
h_{b}^{a} & =\left(\lambda^{-1} \delta \lambda\right)_{b}^{a}=\frac{\delta U}{U}+\frac{1}{U^{2}}\langle\boldsymbol{\Sigma}\rangle, & h_{\rho}^{\rho} & =-2 \frac{\delta U}{U} \\
\lambda^{-1} \lambda^{\prime} & =\frac{U^{\prime}}{U}+\frac{1}{U^{2}}\langle\mathbf{L}\rangle, & \lambda^{\prime} \lambda^{-1} & =\tau^{0}\left[-\frac{U^{\prime}}{U}+\frac{1}{U^{2}}\langle\mathbf{L}\rangle\right] \tau^{0},
\end{aligned}
$$

where $\mathbf{L}$ and $\boldsymbol{\Sigma}$ are defined by

$$
\mathbf{L} \equiv \mathbf{X} \times \mathbf{X}^{\prime}, \quad \mathbf{\Sigma} \equiv \mathbf{X} \times \delta \mathbf{X}
$$

Some other vector representation of geometric quantities and useful vector identities are given in the Appendix B.

For the given metric, the Killing vector is taken as $\xi^{\mu} \equiv\left(k^{a}, 0\right)$, which include $\xi_{T}$ and $\xi_{R}$ as the special cases. For this Killing vector, one obtains

$$
\nabla_{\rho} \xi_{a}=-\nabla_{a} \xi_{\rho}=\frac{1}{2}\left(k \lambda^{\prime}\right)_{a}, \quad \nabla_{a} \xi_{b}=0 .
$$

Now, one can see that the ADT potentials for Einstein-Hilbert term, of which specific component leads to mass and angular momentum, are given by

$$
\begin{aligned}
Q_{R}^{a b} & =0 \\
Q_{R}^{\rho a} & =\frac{\zeta^{2}}{2} U^{2}\left[k \lambda\left(\nabla_{\mu} h_{\rho}^{\mu}+\left(\lambda^{-1} \delta \lambda\right)^{\prime}+2 \frac{\delta U}{U} \lambda^{-1} \lambda^{\prime}\right) \lambda^{-1}\right]^{a} \\
& =\frac{\zeta^{2}}{2}\left[-k\left(\mathbf{X} \cdot \delta \mathbf{X}^{\prime}\right)+k \tau^{0}\langle\delta \mathbf{L}\rangle \tau^{0}\right]^{a}
\end{aligned}
$$

\footnotetext{
${ }^{2}$ From now on, we will drop the identity matrix notation, $\mathbf{1}$ just for notational convenience.
} 
where we used various formulae given in the Appendix B. Note also that

$$
\delta R \equiv \nabla^{\mu} \nabla^{\nu} h_{\mu \nu}-\nabla^{2} h-R^{\mu \nu} h_{\mu \nu}=-\zeta^{2}\left[3 \mathbf{X}^{\prime} \cdot \delta \mathbf{X}^{\prime}+2 \mathbf{X} \cdot \delta \mathbf{X}^{\prime \prime}+2 \delta \mathbf{X} \cdot \mathbf{X}^{\prime \prime}\right] .
$$

Since the vector representation of the ADT potential for $K$ term is somewhat involved, calculational details are relegated to the Appendix C. Collecting all the terms in Appendix $\mathrm{C}$ and using vector identities in the Appendix B, one can obtain the vector representation of the ADT potential for $K$ term and then see that

$$
\begin{aligned}
\eta\left[\sigma Q_{R}^{\rho t}+\frac{1}{m^{2}} Q_{K}^{\rho t}\right]_{\xi_{T}} & =\frac{1}{L}\left[-\frac{\zeta^{2}}{2} \delta J^{2}+\Delta_{C o r}\right] \\
\eta\left[\sigma Q_{R}^{\rho t}+\frac{1}{m^{2}} Q_{K}^{\rho t}\right]_{\xi_{R}} & =\frac{\zeta^{2}}{2} \delta\left(J^{0}-J^{1}\right)
\end{aligned}
$$

where $\mathbf{J}=\left(J^{0}, J^{1}, J^{2}\right)$ is given by

$$
\mathbf{J}=\eta\left(\sigma \mathbf{L}+\frac{\zeta^{2}}{m^{2}}\left[2 \mathbf{L} \times \mathbf{L}^{\prime}+\mathbf{X}^{2} \mathbf{L}^{\prime \prime}+\frac{1}{8}\left(\mathbf{X}^{\prime 2}-4 \mathbf{X} \cdot \mathbf{X}^{\prime \prime}\right) \mathbf{L}\right]\right) .
$$

The above $\mathbf{J}$ vector is the so-called super angular momentum in NMG, and $\Delta_{C o r}$ is the so-called correction term to mass. The correction term to mass is composed of two parts, one of which comes from Einstein-Hilbert term and the other from $K$-term as

$$
\Delta_{C o r}=\Delta_{R}+\Delta_{K}
$$

Each term is given by

$$
\begin{aligned}
\Delta_{R}=\eta \sigma \frac{\zeta^{2}}{2} & \left.-\left(\mathbf{X} \cdot \delta \mathbf{X}^{\prime}\right)\right] \\
\Delta_{K}=\eta \frac{\zeta^{4}}{m^{2}}[ & -U^{2}\left(\mathbf{X}^{\prime \prime} \cdot \delta \mathbf{X}^{\prime}\right)+\frac{U^{2}}{2}\left[(U \delta U)^{\prime \prime \prime}-\left(\mathbf{X} \cdot \delta \mathbf{X}^{\prime}\right)^{\prime \prime}-\frac{1}{2}\left(\mathbf{X}^{\prime} \cdot \delta \mathbf{X}^{\prime}\right)^{\prime}\right] \\
& -\frac{U U^{\prime}}{4}\left[(U \delta U)^{\prime \prime}-\frac{5}{2}\left(\mathbf{X}^{\prime} \cdot \delta \mathbf{X}^{\prime}\right)\right]+\left[\mathbf{X}^{\prime 2}-\left(U U^{\prime}\right)^{\prime}\right](U \delta U)^{\prime}+U U^{\prime}\left(\mathbf{X}^{\prime \prime} \cdot \delta \mathbf{X}\right) \\
& \left.+\left[\frac{5}{4}\left(U U^{\prime}\right)^{\prime}-\frac{21}{16} \mathbf{X}^{\prime 2}\right]\left(\mathbf{X} \cdot \delta \mathbf{X}^{\prime}\right)+\left[-\frac{1}{2}\left(U U^{\prime}\right)^{\prime \prime}+\frac{9}{4}\left(\mathbf{X}^{\prime} \cdot \mathbf{X}^{\prime \prime}\right)\right] U \delta U\right] .
\end{aligned}
$$

Finally, mass and angular momentum in NMG can be obtained by

$$
\begin{aligned}
M & =\frac{1}{4 G} \sqrt{-\operatorname{det} g} \eta\left[\sigma Q_{R}^{r t}+\frac{1}{m^{2}} Q_{K}^{r t}\right]_{\xi_{T}, r \rightarrow \infty} \\
J & =\frac{1}{4 G} \sqrt{-\operatorname{det} g} \eta\left[\sigma Q_{R}^{r t}+\frac{1}{m^{2}} Q_{K}^{r t}\right]_{\xi_{R}, r \rightarrow \infty}
\end{aligned}
$$

The above super angular momentum has been obtained by integrating the EOM of NMG once, as 20 ]

$$
\mathbf{J}=\mathbf{L}-\frac{\zeta^{2}}{m^{2}}\left[\mathbf{X}^{2}\left(\mathbf{X} \times \mathbf{X}^{\prime \prime \prime}-\mathbf{X}^{\prime} \times \mathbf{X}^{\prime \prime}\right)+2\left(\mathbf{X} \cdot \mathbf{X}^{\prime}\right) \mathbf{L}^{\prime}+\left(\frac{1}{8} \mathbf{X}^{\prime 2}-\frac{5}{2} \mathbf{X} \cdot \mathbf{X}^{\prime \prime}\right) \mathbf{L}\right],
$$

which is identical with the above form of the super angular momentum under the choic ${ }^{3}$ of $\eta=$ $\sigma=-1$ as one can verify from the vector identities in the Appendix B.

\footnotetext{
${ }^{3}$ This is the sign choice in [20]. From now on, this sign choice is assumed for the $W A d S_{3}$ case.
} 


\subsection{Mass and Angular momentum of BTZ and Warped $A d S_{3}$ Black Holes}

Through the $S L(2, \mathbf{R})$ reduction, black hole solutions may be represented in terms of a $S O(1,2)$ vector $\mathbf{X}$. More explicitly, the EOM and Hamiltonian constraint in the NMG case given as (2.14) and (2.15) in [20] can be solved by a suitable choice of a vector $\mathbf{X}$, which in turn leads to black hole solutions. Since BTZ and three dimensional warped $A d S\left(W A d S_{3}\right)$ black holes are already obtained in this way [6] [5], we give their corresponding vectors in the following and then present their mass and angular momentum.

Using $S O(1,2)$ vectors corresponding BTZ and $W A d S_{3}$ black holes, we present mass and angular momentum for each black holes. First, BTZ black holes can be represented by

$$
\mathbf{X}=\rho \boldsymbol{\eta}+\boldsymbol{\xi} .
$$

where

$$
\boldsymbol{\eta}=\left(0,-L^{2}, 0\right), \quad \boldsymbol{\xi}=\left(\frac{L^{2}}{2}\left(\rho_{+}+\rho_{-}\right), \frac{L^{2}}{2}\left(\rho_{+}+\rho_{-}\right), L^{2} \sqrt{\rho_{+} \rho_{-}}\right)
$$

This vector $\mathbf{X}$ with $\zeta^{2}=4 / L^{6}$ leads to BTZ metric as

$$
d s^{2}=L^{2}\left[-\frac{\left(\rho-\rho_{+}\right)\left(\rho-\rho_{-}\right)}{\rho} d t^{2}+\rho\left(d \phi+\frac{\sqrt{\rho_{+} \rho_{-}}}{\rho} d t\right)^{2}+\frac{d \rho^{2}}{4\left(\rho-\rho_{+}\right)\left(\rho-\rho_{-}\right)}\right],
$$

and super angular momentum as

$$
\mathbf{J}=\eta\left[\sigma+\frac{\zeta^{2}}{8 m^{2}} \boldsymbol{\eta}^{2}\right](\boldsymbol{\xi} \times \boldsymbol{\eta})=\eta\left(\sigma+\frac{1}{2 m^{2} L^{2}}\right)\left[L^{4} \sqrt{\rho_{+} \rho_{-}} \boldsymbol{\epsilon}_{1}-\frac{L^{4}}{2}\left(\rho_{+}+\rho_{-}\right) \boldsymbol{\epsilon}_{2}\right],
$$

where $\boldsymbol{\epsilon}_{1}=(1,0,0)$ and $\boldsymbol{\epsilon}_{2}=(0,0,1)$. One may note that EOM and Hamiltonian constraint lead to $L^{2}=l^{2} / 2 \cdot\left(\sigma \pm \sqrt{1-1 / m^{2} l^{2}}\right)$ in this case.

To obtain $W A d S_{3}$ black holes, one takes the $S O(1,2)$ vector $\mathbf{X}$ as

$$
\mathbf{X}=\rho^{2} \boldsymbol{\alpha}+\rho \boldsymbol{\beta}+\boldsymbol{\gamma}
$$

where $\boldsymbol{\alpha}, \boldsymbol{\beta}, \boldsymbol{\gamma}$ can be chosen as

$$
\boldsymbol{\alpha}=\left(\frac{1}{2},-\frac{1}{2}, 0\right), \quad \boldsymbol{\beta}=(\omega,-\omega,-1), \quad \gamma=2 u \boldsymbol{\alpha}+\left(1-\beta^{2}\right)\left(\frac{1}{2}, \frac{1}{2},-\omega\right) .
$$

Here, $u$ is defined as $2 u \equiv\left(1-\beta^{2}\right) \omega^{2}+\frac{\beta^{2} \rho_{0}^{2}}{1-\beta^{2}}$. These vectors form a $S O(1,2)$ basis and satisfy the following properties

$$
\begin{aligned}
& \boldsymbol{\alpha}^{2}=0, \quad \boldsymbol{\beta}^{2}=1, \quad \boldsymbol{\gamma}^{2}=-\beta^{2} \rho_{0}^{2}, \quad \boldsymbol{\alpha} \cdot \boldsymbol{\beta}=0, \quad \boldsymbol{\alpha} \cdot \boldsymbol{\gamma}=-\frac{1}{2}\left(1-\beta^{2}\right), \quad \boldsymbol{\beta} \cdot \boldsymbol{\gamma}=0, \\
& \boldsymbol{\alpha} \times \boldsymbol{\beta}=-\boldsymbol{\alpha}, \quad \boldsymbol{\beta} \times \boldsymbol{\gamma}=\frac{2 \beta^{2} \rho_{0}^{2}}{1-\beta^{2}} \boldsymbol{\alpha}-\boldsymbol{\gamma}, \quad \boldsymbol{\gamma} \times \boldsymbol{\alpha}=\frac{1}{2}\left(1-\beta^{2}\right) \boldsymbol{\beta} .
\end{aligned}
$$

\footnotetext{
${ }^{4}$ This is not unique choice for two vectors, since one may perform $S O(1,2)$ transformation to these.
} 
This $S O(1,2)$ vector $\mathbf{X}$ leads to the $W A d S_{3}$ black holes metric as [3] 20]

$$
d s^{2}=-\frac{\beta^{2}\left(\rho^{2}-\rho_{0}^{2}\right)}{Z^{2}} d t^{2}+\frac{d \rho^{2}}{\zeta^{2} \beta^{2}\left(\rho^{2}-\rho_{0}^{2}\right)}+Z^{2}\left(d \phi-\frac{\rho+\left(1-\beta^{2}\right) \omega}{Z^{2}} d t\right)^{2},
$$

where $Z^{2}$ is defined by

$$
Z^{2}=\rho^{2}+2 \omega \rho+\left(1-\beta^{2}\right) \omega^{2}+\frac{\beta^{2} \rho_{0}^{2}}{1-\beta^{2}}
$$

and to satisfy the Hamiltonian constraint in this case, $\zeta$ should be taken as $\zeta^{2} / m^{2}=8 /\left(21-4 \beta^{2}\right)$. Note that for this vector $\mathbf{X}$ one obtains

$$
\mathbf{L}=\left(\rho^{2}-\frac{2 \beta^{2} \rho_{0}^{2}}{1-\beta^{2}}\right) \boldsymbol{\alpha}+\left(1-\beta^{2}\right) \rho \boldsymbol{\beta}+\boldsymbol{\gamma}, \quad \mathbf{L} \times \mathbf{L}^{\prime}=\left(1-\beta^{2}\right) \mathbf{X} .
$$

The background metric and the related basis vectors $\boldsymbol{\alpha}_{B}, \boldsymbol{\beta}_{B}, \boldsymbol{\gamma}_{B}$ can also be defined by setting $\omega=\rho_{0}=0$ in the above expression and, satisfies the similar properties.

Before presenting the result about mass and angular momentum of BTZ and $W A d S_{3}$ black holes in NMG, we need some remarks to clarify the super angular momentum approach focusing on the BTZ case of pure Einstein-Hilbert gravity with cosmological constant. The super angular momentum of pure Einstein-Hilbert gravity is simply given by

$$
\mathbf{L}=\mathbf{X} \times \mathbf{X}^{\prime} .
$$

Since this is obtained by integrating EOM once in the vector representation and can be shown to be conserved, in general it may contain the non-linear effects. That is to say, at the non-linear level, conserved charges are related to subtraction of the background values from the black hole ones

$$
\Delta \mathbf{L} \equiv \mathbf{L}-\mathbf{L}_{B}=\Delta \mathbf{X} \times \mathbf{X}_{B}^{\prime}+\mathbf{X}_{B} \times \Delta \mathbf{X}^{\prime}+\Delta \mathbf{X} \times \Delta \mathbf{X}^{\prime}
$$

Since $\mathbf{X}$ is already linear in some sense, the linearized version of this subtraction in super angular momentum, which will be denoted as $\delta \mathbf{L}$, may be taken as the sum of the first two terms in the above expression for $\Delta \mathbf{L}$. Incidentally in the BTZ case, the last term vanishes automatically and there is no difference between linear and non-linear version. Noting that the contribution of correction terms to mass vanishes in the case of BTZ black holes, one can see that mass and angular momentum of BTZ black holes are given by

$$
M=\frac{\rho_{+}+\rho_{-}}{8 G} \eta\left(\sigma+\frac{1}{2 m^{2} L^{2}}\right), \quad J=\frac{L \sqrt{\rho_{+} \rho_{-}}}{4 G} \eta\left(\sigma+\frac{1}{2 m^{2} L^{2}}\right),
$$

which is consistent with the results from other methods [33]

However, the situation is different in the case of $W A d S_{3}$ black holes. To get the correct angular momentum in this case, one should consider the full non-linear effects. Therefore, one should elevate the expression of the ADT potential for angular momentum in Eq. (34) to the non-linear subtraction form. Concretely, the angular momentum expression is read as

$$
J=\frac{1}{4 G} \sqrt{-\operatorname{det} g}\left[\frac{\zeta^{2}}{2} \Delta\left(J^{0}-J^{1}\right)\right] .
$$


It is interesting to note that this procedure has no effect on mass computation. As we will see in the next section, it turns out that even mass needs non-linear correction in new type black holes.

One way to understand the necessity of this non-linear completion for angular momentum and its irrelevance for mass in the case of $W A d S_{3}$ black holes is to pay attention to the contribution from Einstein-Hilbert term and $K$-term separately in the Lagrangian. Though each contribution to the angular momentum from the Einstein-Hilbert term and the $K$-term is divergent when $\rho$ goes to the infinity, the sum of them is not divergent and gives us the next leading finite contribution, whereas this is not the case for mass since each contribution is finite. Recalling that only the linear metric deviation $h_{\mu \nu}$ is captured in our formalism of the ADT potential, the cancelation of the leading contribution of the ADT potential indicates strongly that it may be insufficient to consider only the linear level contribution. Specifically, the quadratic order contribution in $h_{\mu \nu}$ would leads some corrections to the finite result of angular momentum. Without resorting to another formalism, one can obtain the correct value of angular momentum through super angular momentum, since super angular momentum is the complete non-linear integrated charges.

Taking into account the previous remark and noting

$$
\Delta \boldsymbol{\alpha}=0, \quad \Delta \boldsymbol{\beta}=2 \omega \boldsymbol{\alpha}, \quad \Delta \boldsymbol{\gamma}=2\left(u-\left(1-\beta^{2}\right) \omega^{2}\right) \boldsymbol{\alpha}+\left(1-\beta^{2}\right) \omega \boldsymbol{\beta}
$$

one obtains

$$
\begin{aligned}
\Delta \mathbf{J} & =\Delta \mathbf{L}-\frac{\zeta^{2}}{m^{2}}\left[2\left(1-\beta^{2}\right) \Delta \mathbf{X}-2 \beta^{2} \rho_{0}^{2} \boldsymbol{\alpha}+\frac{1}{8}\left(5-4 \beta^{2}\right) \Delta \mathbf{L}\right] \\
& =\frac{16}{21-4 \beta^{2}}\left[-\left(\beta^{2}\left(1-\beta^{2}\right) \omega^{2}+\frac{\beta^{2} \rho_{0}^{2}}{1-\beta^{2}}\right) \boldsymbol{\alpha}+\beta^{2}\left(1-\beta^{2}\right) \omega \boldsymbol{\beta}\right] \\
& =\frac{16}{21-4 \beta^{2}}\left[\beta^{2}\left(\left(1-\beta^{2}\right) \omega^{2}-\frac{\rho_{0}^{2}}{1-\beta^{2}}\right) \boldsymbol{\alpha}_{B}+\beta^{2}\left(1-\beta^{2}\right) \omega \boldsymbol{\beta}_{B}\right]
\end{aligned}
$$

and the correction terms to mass are given by

$$
\Delta_{R}=\frac{\zeta^{2}}{2}\left(1-\beta^{2}\right) \omega, \quad \Delta_{K}=-\frac{\zeta^{4}}{16 m^{2}}\left(21-20 \beta^{2}\right)\left(1-\beta^{2}\right) \omega .
$$

Finally, mass of $W A d S_{3}$ black holes is given by

$$
M=\frac{4 \zeta \beta^{2}\left(1-\beta^{2}\right) \omega}{G\left(21-4 \beta^{2}\right)}
$$

and their angular momentum by

$$
J=\frac{2 \beta^{2} \zeta}{G\left(21-4 \beta^{2}\right)}\left[\left(1-\beta^{2}\right) \omega^{2}-\frac{\rho_{0}^{2}}{1-\beta^{2}}\right] .
$$

This result about angular momentum is already in [20]. But, mass is obtained by assuming the validity of the first law of black hole thermodynamics or through $A d S / C F T$ correspondence not as conserved charge. Our explicit computation of mass as conserved charge in Eq. (52) through Eq. (33) verify the first law of black hole thermodynamics and checks $A d S / C F T$ correspondence in this case. Note that the correction term to mass gives us exactly the same value with the super 
angular momentum and mass becomes twice of the value given by super angular momentum. This result confirms the form given in [20] 33.

For comparison, let us present the linearized version of $\Delta \mathbf{J}$, denoted as $\delta \mathbf{J}$

$\delta \mathbf{J}=\delta \mathbf{L}-\frac{\zeta^{2}}{8 m^{2}}\left[\left\{2\left(1-\beta^{2}\right)\left(21-4 \beta^{2}\right) \omega \rho-\left(5-4 \beta^{2}\right)\left(\left(1-\beta^{2}\right) \omega^{2}+\frac{\beta^{2} \rho_{0}^{2}}{1-\beta^{2}}\right)\right\} \boldsymbol{\alpha}_{B}+\left(21-20 \beta^{2}\right)\left(1-\beta^{2}\right) \omega \boldsymbol{\beta}_{B}\right]$.

As can be seen easily, the nonlinear effects in $\mathbf{J}$ come from $\Delta \mathbf{L}$ and $\Delta \mathbf{X}^{2}$.

\section{The Conserved Charges of New Type Black Holes}

In this section we confine ourselves to the simplest version of new type black holes: static nonrotating ones. Though the mass of new type black holes are already computed in the linearized ADT formalism [24] 25], it is inconsistent with $A d S / C F T$ correspondence as was shown in [33]. We reexamine mass of new type black holes as conserved charges by using the formalism presented in the previous section. As the case of $W A d S_{3}$ black holes, in which the ADT formalism needs to be supplemented by super angular momentum to obtain correct angular momentum of black holes, it turns out that even mass needs non-linear corrections in the case of new type black holes. Therefore, we rederive new type black hole solutions in the $S L(2, \mathbf{R})$ reduction formalism, and then present our results about mass of new type black holes.

In order to get the new type black hole solution in $S L(2, \mathbf{R})$ reduction formalism, we can take the vector ansatz as a form

$$
\mathbf{X}=\rho \boldsymbol{\eta}+\sqrt{\rho} \boldsymbol{\kappa}+\boldsymbol{\xi} .
$$

For the above vectors, we need to impose the following conditions

$$
\boldsymbol{\kappa}^{2}=0, \quad \boldsymbol{\xi}^{2}=0, \quad \boldsymbol{\kappa} \cdot \boldsymbol{\xi}=0, \quad(\boldsymbol{\eta} \cdot \boldsymbol{\kappa}) \boldsymbol{\xi}-(\boldsymbol{\eta} \cdot \boldsymbol{\xi}) \boldsymbol{\kappa}=0
$$

which can be satisfied by taking

$$
\boldsymbol{\eta}=\left(0,-L^{2}, 0\right), \boldsymbol{\kappa}=\left(-\frac{b}{2} L^{2},-\frac{b}{2} L^{2}, 0\right), \boldsymbol{\xi}=\left(-\frac{c}{2} L^{2},-\frac{c}{2} L^{2}, 0\right) .
$$

EOM and Hamiltonian constraint are given by

$$
\left(\boldsymbol{\eta}^{2}-8 \sigma \frac{m^{2}}{\zeta^{2}}\right) \boldsymbol{\kappa}=0, \quad \frac{1}{32}\left(\boldsymbol{\eta}^{2}\right)^{2}-\sigma \frac{m^{2}}{2 \zeta^{2}} \boldsymbol{\eta}^{2}+\frac{2 m^{2}}{\zeta^{4} l^{2}}=0,
$$

which lead to $\zeta^{2}=8 m^{2} / L^{4}=4 / L^{6}$ and $l^{2}=2 L^{2}$, respectively. It turns out that the sign choice of $\eta=\sigma=1$ is necessary for the existence of new type black holes. The above vector $\mathbf{X}$ leads to the metric of new type black holes in the form of

$$
d s^{2}=L^{2}\left[-(\rho+b \sqrt{\rho}+c) d t^{2}+\rho d \phi^{2}+\frac{d \rho^{2}}{4 \rho(\rho+b \sqrt{\rho}+c)}\right] .
$$


with $\zeta^{2}=4 / L^{6}$. By the coordinate transformation $\rho=r^{2}$, new type black hole solutions become

$$
d s^{2}=L^{2}\left[-\left(r^{2}+b r+c\right) d t^{2}+\frac{d r^{2}}{\left(r^{2}+b r+c\right)}+r^{2} d \phi^{2}\right],
$$

which is the form given in [16].

To obtain mass of new type black holes, we compute the super angular momentum and correction terms to mass. By inserting the above three vectors $\boldsymbol{\eta}, \boldsymbol{\kappa}$ and $\boldsymbol{\xi}$ into the formula (35), we obtain super angular momentum in the form of

$$
\mathbf{J}=\boldsymbol{\xi} \times \boldsymbol{\eta}+\frac{\zeta^{2}}{8 m^{2}}\left[\boldsymbol{\eta}^{2} \boldsymbol{\xi} \times \boldsymbol{\eta}+(\boldsymbol{\eta} \cdot \boldsymbol{\kappa})(\boldsymbol{\eta} \times \boldsymbol{\kappa})\right]=\left[c-\frac{1}{4} b^{2}\right] L^{4} \boldsymbol{\epsilon}_{2}+\mathcal{O}\left(\frac{1}{\sqrt{\rho}}\right) .
$$

As can be shown from (37) and (38), correction terms to the mass in new type black holes are given by

$$
\Delta_{R}=-\frac{\zeta^{2}}{2} \frac{L^{4} b}{4} \sqrt{\rho}=-\frac{b}{2 L^{2}} \sqrt{\rho}, \quad \Delta_{K}=\frac{\zeta^{4}}{m^{2}} \frac{L^{8} b}{64} \sqrt{\rho}=\frac{b}{2 L^{2}} \sqrt{\rho} .
$$

Note that the total contribution of correction terms to mass vanishes. Finally, one can see that mass of new type black holes is given by

$$
M=\frac{1}{4 \zeta G L}\left[-\frac{\zeta^{2}}{2} \Delta J^{2}\right]=\frac{b^{2}-4 c}{16 G},
$$

which is consistent with the result by $A d S / C F T$ correspondence and satisfies the simple form of the first law of black hole thermodynamics as [33] (See [31] for the extension to the the case of rotating new type black holes)

$$
d M=T d S_{B H} .
$$

This shows us that $A d S / C F T$ correspondence may be used as a guiding principle to obtain physical quantities for gravity. As we have already seen in the above results, there is no non-linear effect in the mass correction terms. The non-linear contribution comes only from the super angular momentum.

\section{Conclusion}

In this paper we have obtained mass and angular momentum, as conserved charges, of several black holes in the so-called new massive gravity(NMG) in three dimension and confirmed the validity of first law of black hole thermodynamics and/or $A d S / C F T$ correspondence in this case. At first, we obtained the ADT potential for the scalar curvature square term and Ricci tensor square one in arbitrary dimension. This leads to the ADT potential for the NMG case, which is our main interest. Then we obtained conserved charges of various black hole solutions, which have at least two commuting Killing vectors. To obtain conserved charges consistent with the first law of black hole thermodynamics and/or AdS/CFT correspondence, we have used super angular momentum method in the $S O(1,2)$ reduction approach supplemented by the ADT formalism, which is developed by Clement 35] 3] 6]. In the following, several comments are in order. 
It has been well known that conserved charges in gravity with higher derivatves are difficult to define. There are attempts called the ADT formalism to accomplish this goal [26] [28], which may be regarded as a natural generalization of the famous ADM formalism. Applying this linear ADT formalism, some charges consistent with the first law of black hole thermodynamics are computed and presented in the Appendix D. Though this approach is covariant, this basically uses the linearized peturbation around the given background and so might be incomplete.

On the other hand, especially in three dimensional case, there is the so-called super angular momentum method [35] 3] which is not fully covariant but may capture non-linear effects. The nature of super angular momentum as the non-linear conserved quantity comes from the fact that it is obtained by the first intergral of equations of motion without perturbative expansion. Though super angular momentum leads to correct results in some cases, it turned out to be inconsistent with the first law of black hole thermodynamics in the case of warped $A d S_{3}$ black holes in TMG, and then it was shown that there is a way to overcome this difficulty by supplementing super angular momentum with the ADT formalism [6] [20. That is to say, by comparing the ADT charges with the linearized super angular momentum a linear correction term to mass was shown to exist in the super angular momentum side. Though there is no a priori guarantee for the absence of non-linear effects in the correction term, it was sufficient for the purpose.

After the advent of $A d S / C F T$ correspondence there are approaches using boundary theory [29] [33] 32] 31] to define the bulk $A d S$ conserved charges. Though this approach is covariant and can capture non-linear effects, it is applicable only to asymptotically (warped) $A d S$ space. Furthermore, one may need some additional information to define conserved charges in this approach and it is difficult to apply this method to some cases, for instance, to warped $A d S_{3}$ black holes without $a$ priori inputs.

In this paper we have adopted Clement's hybrid formalism of super angular momentum method and ADT approach to obtain mass and angular momentum of various black holes in the NMG case. This hybrid formalism gives us results consistent with the first law of black hole thermodynamics in all the cases considered in this paper. Since $A d S / C F T$ approach leads to the same results with ours in all the cases, our results can be regared as consistent with $A d S / C F T$ correspondence.

In the present state of things there are no completely satisfactory covariant non-linear method to define conserved charges in general. Several approaches have their own merits and demerits as was commented in the above. It will be very interesting to improve this situation and resolve some issues, for example, whether or not the non-linear effects exist generically for the mass correction term in the hybrid formalism.

\section{Acknowledgements}

This work was supported by the National Research Foundation of Korea(NRF) grant funded by the Korea government(MEST) through the Center for Quantum Spacetime(CQUeST) of Sogang 
University with grant number 2005-0049409. S.H.Y would like to thank Prof. Seungjoon Hyun at Yonsei University for discussion and encouragement. S.H.Y was supported in part by the National Research Foundation of Korea (NRF) grant funded by the Korea government (MEST) with the grant number 2009-0085995. This work of S.N and J.D.P was supported by a grant from the Kyung Hee University in 2009(KHU-20100130). S.N was also supported by the National Research Foundation of Korea(NRF) grant funded by the Korean government(MEST)(No. 2009-0063068). 


\section{APPENDICES}

In this appendix, some calculational details are given. In section $A$, we present some details for ADT charge computation and in section $B$ give various formulae for vector calculation. In section $C$, some details about vector representation of the ADT potential are given and the ADT potential in Schwarzschild coordinates are given in the appendix D.

\section{A The Formulae for the ADT Potential Computation}

Some Killing vector properties:

Our curvature convention is

$$
\left[\nabla_{\alpha}, \nabla_{\beta}\right] V^{\mu}=-R_{\nu \alpha \beta}^{\mu} V^{\nu}
$$

which implies for a scalar $S$

$$
\left[\nabla_{\mu}, \nabla^{2}\right] S=\left[\nabla_{\mu}, \nabla_{\nu}\right] \nabla^{\nu} S=-R_{\mu \nu} \nabla^{\nu} S .
$$

Covariant derivatives of Killing vectors, $\xi$, satisfy

$$
\nabla_{\mu} \nabla_{\nu} \xi_{\alpha}=-R_{\mu \alpha \nu}^{\beta} \xi_{\beta}, \quad \nabla^{2} \xi^{\mu}=-R_{\nu}^{\mu} \xi^{\nu}
$$

and

$$
\begin{aligned}
\mathcal{L}_{\xi} R & =\xi^{\mu} \nabla_{\mu} R=0 \\
\mathcal{L}_{\xi} \nabla_{\mu} R & =\xi^{\alpha} \nabla_{\alpha} \nabla_{\mu} R+\nabla_{\alpha} R \nabla_{\mu} \xi^{\alpha}=0 \\
\mathcal{L}_{\xi} R_{\mu \nu} & =\xi^{\alpha} \nabla_{\alpha} R_{\mu \nu}+R_{\mu \alpha} \nabla_{\nu} \xi^{\alpha}+R_{\nu \alpha} \nabla_{\mu} \xi^{\alpha}=0 .
\end{aligned}
$$

Bianchi identity and the Killing property lead to the following identity

$$
R_{\mu \alpha \nu \beta} \nabla^{\alpha} \xi^{\beta}=\frac{1}{2} R_{\mu \nu \alpha \beta} \nabla^{\alpha} \xi^{\beta} .
$$

The variation of some quantities

$$
\begin{aligned}
\delta \Gamma_{\mu \nu}^{\alpha}= & \frac{1}{2}\left(\nabla_{\mu} h_{\nu}^{\alpha}+\nabla_{\nu} h_{\mu}^{\alpha}-\nabla^{\alpha} h_{\mu \nu}\right), \\
\nabla^{\mu} \delta \Gamma_{\mu \nu}^{\alpha}= & -g^{\alpha \beta} \delta R_{\nu \beta}-\frac{1}{2} \nabla_{\nu} \nabla^{\alpha} h+\nabla^{\beta} \nabla_{\nu} h_{\beta}^{\alpha}, \\
\delta\left(\nabla^{2} R\right)= & \nabla^{2} \delta R-h_{\alpha \beta} \nabla^{\alpha} \nabla^{\beta} R-g^{\alpha \beta} \delta \Gamma_{\alpha \beta}^{\nu} \nabla_{\nu} R \\
= & \nabla^{2} \delta R-\nabla^{\alpha}\left(h_{\alpha \beta} \nabla^{\beta} R\right)+\frac{1}{2} \nabla^{\alpha} h \nabla_{\alpha} R, \\
\delta\left(\nabla_{\mu} \nabla_{\nu} R\right)= & \nabla_{\mu} \nabla_{\nu} \delta R-\delta \Gamma_{\mu \nu}^{\alpha} \nabla_{\alpha} R, \\
\delta\left(\nabla^{2} R_{\mu \nu}\right)= & \nabla^{2} \delta R_{\mu \nu}-h_{\alpha \beta} \nabla^{\alpha} \nabla^{\beta} R_{\mu \nu}-g^{\alpha \beta} \delta \Gamma_{\alpha \beta}^{\nu} \nabla_{\nu} R_{\mu \nu} \\
& -\nabla^{\alpha}\left(\delta \Gamma_{\alpha \mu}^{\beta} R_{\beta \nu}\right)-\nabla^{\alpha}\left(\delta \Gamma_{\alpha \nu}^{\beta} R_{\beta \mu}\right)-\delta \Gamma_{\alpha \mu}^{\beta} \nabla^{\alpha} R_{\beta \nu}-\delta \Gamma_{\alpha \nu}^{\beta} \nabla^{\alpha} R_{\beta \mu}, \\
\delta\left(R_{\alpha \beta} R^{\alpha \beta}\right)= & 2 h^{\alpha \beta} R_{\rho \alpha \beta \sigma} R^{\rho \sigma}+R_{\alpha \beta}\left[\nabla^{\alpha} \nabla_{\gamma} h^{\gamma \beta}+\nabla^{\beta} \nabla_{\gamma} h^{\gamma \alpha}-\nabla^{\alpha} \nabla^{\beta} h-\nabla^{2} h^{\alpha \beta}\right] .
\end{aligned}
$$


Some formulae for $Q_{R_{2}}^{\mu \nu}$ computation:

Each $E_{a}^{\mu}(a=1,2, \cdots, 5)$ term is computed separately, as follows. First, let us consider $E_{1}^{\mu}$ term. The same term may be subtracted from and added to $E_{1}^{\mu}$ just for our calculational convenience. Then, the $E_{1}^{\mu}$ term becomes

$$
\begin{aligned}
E_{1}^{\mu}= & -h^{\mu \alpha} R_{\alpha \rho \sigma \nu} R^{\rho \sigma} \xi^{\nu}+\left(R_{\alpha \gamma \nu}^{\mu}+2 R_{\gamma \alpha \nu}^{\mu}\right) \xi^{\nu} R_{\beta}^{\gamma} h^{\alpha \beta}+\xi^{\mu} R_{\gamma \alpha} R_{\beta}^{\gamma} h^{\alpha \beta} \\
& +A_{1}^{\mu}-\frac{1}{2} \xi^{\mu} R^{\alpha \beta} \nabla^{2} h_{\alpha \beta}+A_{2}^{\mu}-R^{\mu \alpha} \xi^{\nu}\left(\nabla^{\gamma} \nabla_{\alpha} h_{\gamma \nu}-\nabla^{2} h_{\alpha \nu}\right)-2 \xi^{\nu} \delta \Gamma_{\alpha \nu}^{\beta} \nabla^{\alpha} R_{\beta}^{\mu} \\
& +A_{3}^{\mu}+2 \xi^{\gamma} R_{\gamma \alpha \beta \nu} \nabla^{\nu} \nabla^{[\mu} h^{\alpha]}+\frac{1}{2} \xi^{\mu} R^{\alpha \beta} \nabla_{\alpha} \nabla_{\beta} h,
\end{aligned}
$$

where

$$
\begin{aligned}
A_{1}^{\mu} \equiv & -R^{\alpha \beta} \xi^{\nu}\left(\nabla_{\nu} \nabla^{\mu} h_{\alpha \beta}-\nabla_{\nu} \nabla_{\alpha} h_{\beta}^{\mu}\right)-\xi^{\mu} R^{\alpha \beta}\left(\nabla^{\gamma} \nabla_{\alpha} h_{\gamma \beta}-\nabla^{2} h_{\alpha \beta}\right) \\
= & \nabla_{\nu}\left[2 R^{\alpha \beta} \xi^{[\mu} \nabla^{\nu]} h_{\alpha \beta}-2 R^{\alpha \beta} \xi^{\mu} \nabla_{\alpha} h_{\beta}^{\nu]}\right] \\
& +\xi^{\nu} \nabla_{\nu} R^{\alpha \beta}\left(\nabla^{\mu} h_{\alpha \beta}-\nabla_{\alpha} h_{\beta}^{\mu}\right)+\nabla_{\nu}\left(\xi^{\mu} R^{\alpha \beta}\right)\left(\nabla_{\alpha} h_{\beta}^{\nu}-\nabla^{\nu} h_{\alpha \beta}\right), \\
A_{2}^{\mu} \equiv & -R^{\alpha \beta} \xi^{\nu}\left(\nabla_{\beta} \nabla_{\alpha} h_{\nu}^{\mu}-\nabla_{\beta} \nabla^{\mu} h_{\alpha \nu}\right)+R^{\mu \alpha} \xi^{\nu}\left(\nabla^{\gamma} \nabla_{\alpha} h_{\gamma \nu}-\nabla^{2} h_{\alpha \nu}\right) \\
= & \nabla_{\nu}\left[2 \xi^{\beta} R^{\alpha[\mu} \nabla_{\alpha} h_{\beta}^{\nu]}-2 \xi^{\beta} R^{\alpha[\mu} \nabla^{\nu]} h_{\alpha \beta}\right] \\
& +\nabla_{\nu}\left(\xi^{\beta} R^{\mu \alpha}\right)\left(\nabla^{\nu} h_{\alpha \beta}-\nabla_{\alpha} h_{\beta}^{\nu}\right)+\nabla_{\nu}\left(\xi^{\beta} R^{\nu \alpha}\right)\left(\nabla^{\mu} h_{\alpha \beta}-\nabla_{\alpha} h_{\beta}^{\mu}\right), \\
A_{3}^{\mu} \equiv & -2 R^{\mu \alpha \beta} \xi^{\nu} \delta R_{\alpha \beta}-2 \xi^{\gamma} R_{\gamma \alpha \beta \nu} \nabla^{\nu} \nabla^{[\mu} h^{\alpha]} \\
= & \nabla_{\nu}\left[R^{\mu \nu}{ }_{\alpha \beta} \xi^{\alpha} \nabla_{\gamma} h^{\gamma \beta}-2 \xi^{\gamma} R_{\gamma}^{\alpha \beta[\mu} \nabla_{\alpha} h_{\beta}^{\nu]}+2 \xi^{\gamma} R_{\gamma}^{\alpha \beta[\mu} \nabla^{\nu]} h_{\alpha \beta}\right] \\
& +\nabla_{\nu}\left(\xi^{\gamma} R_{\gamma}^{\alpha \beta \mu}\right)\left(\nabla_{\alpha} h_{\beta}^{\nu}-\nabla^{\nu} h_{\alpha \beta}\right)+\nabla_{\nu}\left(\xi^{\gamma} R_{\gamma}^{\alpha \beta \nu}\right)\left(\nabla^{\mu} h_{\alpha \beta}-\nabla_{\alpha} h_{\beta}^{\mu}\right) \\
& +\xi^{\nu}\left(\nabla_{\nu} R_{\beta}^{\mu}-\nabla_{\beta} R_{\nu}^{\mu}\right) \nabla_{\alpha} h^{\alpha \beta}-R_{\rho \sigma \beta}^{\mu} \nabla^{\rho} \xi^{\sigma} \nabla_{\alpha} h^{\alpha \beta} \\
& -R_{\gamma \alpha \nu}^{\mu} \xi^{\nu} R_{\beta}^{\gamma} h^{\alpha \beta}-R_{\rho \sigma \nu}^{\mu} \xi^{\nu} R^{\sigma \alpha \beta} h_{\alpha \beta}-R_{\alpha \beta \nu}^{\mu} \xi^{\nu} \nabla^{\alpha} \nabla^{\beta} h .
\end{aligned}
$$

For $A_{1}^{\mu}$ and $A_{2}^{\mu}$, one can see that

$$
\begin{aligned}
A_{1}^{\mu} & +A_{2}^{\mu}-2 \xi^{\nu} \delta \Gamma_{\alpha \nu}^{\beta} \nabla^{\alpha} R_{\beta}^{\mu} \\
= & \nabla_{\nu}\left[2 \xi^{\alpha} R^{\beta[\mu} \nabla_{\beta} h_{\alpha}^{\nu]}-2 \xi^{\alpha} R^{\beta[\mu} \nabla^{\nu]} h_{\alpha \beta}+2 R^{\alpha \beta} \xi^{[\mu} \nabla^{\nu]} h_{\alpha \beta}-2 R^{\alpha \beta} \xi^{[\mu} \nabla_{\alpha} h_{\beta}^{\nu]}+2 h^{\alpha \beta} \nabla_{\alpha}\left(\xi^{[\mu} R_{\beta}^{\nu]}\right)\right] \\
& -\frac{1}{2} \xi^{\nu} \nabla^{\alpha} R\left(\nabla^{\mu} h_{\alpha \nu}-\nabla_{\alpha} h_{\nu}^{\mu}\right)-R_{\alpha \nu} \nabla^{\nu} \xi^{\beta}\left(\nabla^{\mu} h_{\beta}^{\alpha}-\nabla^{\alpha} h_{\beta}^{\mu}\right) \\
& +\xi^{\gamma} \nabla_{\gamma} R^{\alpha \beta}\left(\nabla^{\mu} h_{\alpha \beta}-\nabla_{\alpha} h_{\beta}^{\mu}\right)-\nabla_{\nu}\left(\xi^{\mu} R^{\alpha \beta}\right) \nabla^{\nu} h_{\alpha \beta}-h^{\alpha \beta} \nabla_{\nu} \nabla_{\beta}\left(\xi^{\mu} R_{\alpha}^{\nu}-\xi^{\nu} R_{\alpha}^{\mu}\right) .
\end{aligned}
$$

For $A_{3}^{\mu}$, one may note that the following identities

$$
\begin{aligned}
\nabla_{\nu}\left(\xi^{\gamma} R_{\gamma}^{\alpha \beta \mu}\right)\left(\nabla_{\alpha} h_{\beta}^{\nu}-\nabla^{\nu} h_{\alpha \beta}\right)= & \nabla_{\nu}\left(h_{\alpha \beta} R_{\gamma}^{\alpha \beta \nu} \nabla^{\mu} \xi^{\gamma}\right)-h^{\alpha \beta} \nabla_{\alpha} R_{\beta \gamma} \nabla^{\mu} \xi^{\gamma}-h^{\alpha \beta} \nabla_{\gamma} R_{\alpha \beta} \nabla^{\gamma} \xi^{\mu} \\
& +R_{\rho \sigma \nu}^{\mu} \xi^{\nu} R^{\sigma \alpha \beta \rho} h_{\alpha \beta}+R_{\alpha \nu \beta}^{\mu} \nabla^{\nu} \xi^{\gamma}\left(\nabla^{\alpha} h_{\gamma}^{\beta}-\nabla^{\beta} h_{\gamma}^{\alpha}\right)
\end{aligned}
$$




$$
\begin{aligned}
\nabla_{\nu}\left(\xi^{\gamma} R_{\gamma}^{\alpha \beta \nu}\right)\left(\nabla^{\mu} h_{\alpha \beta}-\nabla_{\alpha} h_{\beta}^{\mu}\right)= & \xi^{\gamma}\left(\nabla_{\alpha} R_{\beta \gamma}-\nabla_{\gamma} R_{\alpha \beta}\right)\left(\nabla^{\mu} h^{\alpha \beta}-\nabla^{\alpha} h^{\mu \beta}\right)-R_{\gamma \alpha \beta \nu} \nabla^{\nu} \xi^{\gamma} \nabla^{\alpha} h^{\mu \beta}, \\
h^{\alpha \beta} \nabla_{\nu} \nabla_{\beta}\left(\xi^{\mu} R_{\alpha}^{\nu}-\xi^{\nu} R_{\alpha}^{\mu}\right)= & h^{\alpha \beta}\left(\nabla_{\alpha} R_{\nu}^{\mu} \nabla_{\beta} \xi^{\nu}+\frac{1}{2} \nabla_{\alpha} R \nabla_{\beta} \xi^{\mu}\right)-h^{\alpha \beta} \xi^{\nu}\left(R_{\alpha}^{\mu} R_{\beta \nu}-R_{\alpha \gamma \nu}^{\mu} R_{\beta}^{\gamma}\right) \\
& +\xi^{\mu} h^{\alpha \beta}\left(R_{\gamma \alpha} R_{\beta}^{\gamma}+R_{\alpha \rho \sigma \beta} R^{\rho \sigma}+\frac{1}{2} \nabla_{\alpha} \nabla_{\beta} R\right) .
\end{aligned}
$$

Collecting various expression in the above, one can obtains $E_{1}^{\mu}$.

For the calculation of $E_{2}^{\mu}$ term, one may note that

$$
\nabla^{2} \nabla_{\nu} Q_{R}^{\mu \nu}=\nabla_{\nu}\left[\nabla^{2} Q^{\mu \nu}+R_{\alpha \beta}^{\mu \nu} Q^{\alpha \beta}+2 Q^{\alpha[\mu} R_{\alpha}^{\nu]}\right]+R_{\alpha}^{\mu} \nabla_{\nu} Q^{\alpha \nu}
$$

which leads to

$$
\begin{aligned}
\xi^{\nu} \nabla^{2} \mathcal{I}^{\mu}{ }_{\nu}= & \nabla^{2}\left(\mathcal{I}^{\mu}{ }_{\nu} \xi^{\nu}\right)-\mathcal{I}^{\mu}{ }_{\nu} \nabla^{2} \xi^{\nu}-2 \nabla_{\nu} \mathcal{I}_{\alpha}^{\mu} \nabla^{\nu} \xi^{\alpha} \\
= & \nabla^{2}\left(\mathcal{I}^{\mu}{ }_{\nu} \xi^{\nu}\right)-\nabla_{\nu}\left(\delta R \nabla^{[\mu} \xi^{\nu]}\right)-2 g^{\mu \rho} \nabla_{\alpha} \delta R_{\rho \nu} \nabla^{\alpha} \xi^{\nu}+\mathcal{I}_{\alpha}^{\mu} R_{\nu}^{\alpha} \xi^{\nu}+\delta R R_{\nu}^{\mu} \xi^{\nu} \\
= & \nabla_{\nu}\left[\nabla^{2} Q_{R}^{\mu \nu}+R^{\mu \nu}{ }_{\alpha \beta} Q_{R}^{\alpha \beta}+2 R_{\alpha}^{[\mu} Q_{R}^{\nu] \alpha}-\delta R \nabla^{[\mu} \xi^{\nu]}\right] \\
& -2 g^{\mu \rho} \nabla_{\nu} \delta R_{\rho \alpha} \nabla^{\nu} \xi^{\alpha}+\nabla^{2}\left(h^{\mu \beta} R_{\beta \nu} \xi^{\nu}+\frac{1}{2} \xi^{\mu} R^{\rho \sigma} h_{\rho \sigma}-\frac{1}{2} R_{\nu}^{\mu} \xi^{\nu} h\right) \\
& +\left(\mathcal{I}^{\mu}{ }_{\alpha} R_{\nu}^{\alpha}+R_{\alpha}^{\mu} \mathcal{I}_{\nu}^{\alpha}\right) \xi^{\nu}+\delta R R_{\nu}^{\mu} \xi^{\nu}-R_{\alpha}^{\mu}\left(h^{\alpha \beta} R_{\beta \nu} \xi^{\nu}+\frac{1}{2} \xi^{\alpha} R^{\rho \sigma} h_{\rho \sigma}-\frac{1}{2} R_{\nu}^{\alpha} \xi^{\nu} h\right) .
\end{aligned}
$$

Noting that the following formula

$$
\begin{aligned}
-2 g^{\mu \rho} \nabla_{\nu} \delta R_{\rho \alpha} \nabla^{\nu} \xi^{\alpha}= & -2 \nabla_{\nu}\left[\nabla^{\alpha} \xi^{\beta} \nabla_{\alpha} \nabla^{[\mu} h_{\beta}^{\nu]}\right]+\frac{1}{2} R_{\nu \alpha \beta}^{\mu} \nabla^{\alpha} \xi^{\beta} \nabla^{\nu} h-2 \xi^{\gamma} R_{\gamma \alpha \beta \nu} \nabla^{\nu} \nabla^{[\mu} h^{\alpha] \beta} \\
& +R_{\alpha \nu} \nabla^{\nu} \xi^{\beta}\left(\nabla^{\mu} h_{\beta}^{\alpha}-\nabla^{\alpha} h_{\beta}^{\mu}\right)-\nabla_{\gamma}\left(h^{\mu \alpha} R_{\alpha \nu}+R_{\alpha \beta \nu}^{\mu} h^{\alpha \beta}\right) \nabla^{\gamma} \xi^{\nu} \\
& +R_{\alpha \nu \beta}^{\mu} \nabla^{\nu} \xi^{\gamma}\left(\nabla^{\beta} h_{\gamma}^{\alpha}-\nabla^{\alpha} h_{\gamma}^{\beta}\right) \\
& +R_{\alpha \beta \nu}^{\gamma} \nabla^{\beta} \xi^{\alpha}\left(\nabla^{\mu} h_{\gamma}^{\nu}-\nabla_{\nu} h_{\gamma}^{\mu}\right)-\frac{1}{2} R_{\nu \rho \sigma}^{\mu} \nabla_{\alpha} h^{\alpha \beta} \nabla^{\rho} \xi^{\sigma} .
\end{aligned}
$$

and the formula containing the $F_{\mu \nu}$ term in (25)

$$
\begin{aligned}
& F_{\nu}^{\mu} \xi^{\nu}+R_{\alpha \nu} \xi^{\nu} \nabla^{2} h^{\mu \alpha}+\mathcal{I}_{\alpha}^{\mu} R_{\nu}^{\alpha} \xi^{\nu}+R_{\alpha}^{\mu} \mathcal{I}_{\nu}^{\alpha} \xi^{\nu} \\
= & \nabla_{\nu}\left[-2 \xi^{\alpha} R_{\alpha}^{[\mu} \nabla_{\beta} h^{\nu] \beta}+\xi^{\alpha} R_{\alpha}^{[\mu} \nabla^{\nu]} h\right]+\nabla_{\beta}\left(\xi^{\alpha} R_{\alpha}^{\mu}\right) \nabla_{\alpha} h^{\alpha \beta} \\
& +R_{\rho \sigma \alpha}^{\mu} h^{\rho \sigma} R_{\nu}^{\alpha} \xi^{\nu}+h^{\mu \alpha} R_{\alpha \beta} R_{\nu}^{\beta} \xi^{\nu}+R_{\nu}^{\mu} \xi^{\nu} R^{\alpha \beta} h_{\alpha \beta} \\
& +R^{\mu \alpha} \xi^{\nu}\left(\nabla^{\gamma} \nabla_{\alpha} h_{\gamma \nu}-\nabla^{2} h_{\alpha \nu}\right)-\frac{1}{2} R^{\mu \alpha} \xi^{\nu} \nabla_{\nu} \nabla_{\alpha} h+\frac{1}{2} R_{\nu}^{\mu} \xi^{\nu} \nabla^{2} h-\frac{1}{2} \nabla_{\nu}\left(\xi^{\alpha} R_{\alpha}^{\mu}\right) \nabla^{\nu} h,
\end{aligned}
$$

one can obtain $E_{2}$ expression.

Formulae for remaining $E_{a}^{\mu}$ are

$$
\begin{aligned}
E_{3}^{\mu}= & \nabla_{\nu}\left[2 \xi^{\beta} h^{\alpha[\mu} \nabla_{\alpha} R_{\beta}^{\nu]}\right]-h^{\mu \alpha} \xi^{\nu}\left(R_{\alpha \rho \sigma \nu} R^{\rho \sigma}+\frac{1}{2} \nabla_{\alpha} \nabla_{\nu} R+R_{\alpha \beta} R_{\nu}^{\beta}\right) \\
& +h^{\alpha \beta} \nabla_{\alpha} R_{\nu}^{\mu} \nabla_{\beta} \xi^{\nu}-\xi^{\nu} \nabla^{\alpha} R_{\nu}^{\beta}\left(\nabla_{\alpha} h_{\beta}^{\mu}+\nabla^{\mu} h_{\alpha \beta}\right) .
\end{aligned}
$$




$$
\begin{aligned}
E_{4}^{\mu} & =\nabla_{\nu}\left[-\xi^{[\mu} h^{\nu] \alpha} \nabla_{\alpha} R\right]+\frac{1}{2} h^{\alpha \beta} \nabla_{\alpha} R \nabla_{\beta} \xi^{\mu}-\frac{1}{2} h^{\mu \alpha} \xi^{\nu} \nabla_{\alpha} \nabla_{\nu} R+\frac{1}{2} \xi^{\nu} \nabla^{\alpha} R\left(h_{\alpha \nu}^{\mu}-\nabla_{\alpha} h_{\nu}^{\mu}\right) . \\
E_{5}^{\mu} & \equiv \frac{1}{2} \xi^{\nu} \nabla_{\alpha} h \nabla^{\alpha} R_{\nu}^{\mu}+\frac{1}{4} \xi^{\mu} \nabla_{\alpha} h \nabla^{\alpha} R .
\end{aligned}
$$

\section{B Identities and Useful Formulae for Vector Representation}

Ricci tensor and scalar curvature are given by

$$
\begin{aligned}
R_{b}^{a} & =-\frac{\zeta^{2}}{2}\left[\left(U U^{\prime}\right)^{\prime}+\langle\mathbf{L}\rangle^{\prime}\right]_{b}^{a}, \quad R_{\rho}^{\rho}=-\frac{\zeta^{2}}{2}\left[2\left(U U^{\prime}\right)^{\prime}-\mathbf{X}^{\prime 2}\right] \\
R & =-\frac{\zeta^{2}}{2}\left[4\left(U U^{\prime}\right)^{\prime}-\mathbf{X}^{\prime 2}\right]=-\frac{\zeta^{2}}{2}\left[4 \mathbf{X} \cdot \mathbf{X}^{\prime \prime}+3 \mathbf{X}^{\prime 2}\right] .
\end{aligned}
$$

Covariant derivative of Ricci tensors

$$
\begin{aligned}
\nabla_{\rho} R_{\rho}^{\rho} & =\left(R_{\rho}^{\rho}\right)^{\prime}=-\zeta^{2}\left[\left(U U^{\prime}\right)^{\prime \prime}-\mathbf{X}^{\prime} \cdot \mathbf{X}^{\prime \prime}\right] \\
\nabla_{\rho} R_{a}^{b} & =\left(R_{a}^{b}\right)^{\prime}+\frac{1}{2}\left(\lambda^{-1} \lambda^{\prime}\right)_{c}^{b} R_{a}^{c}-\frac{1}{2} R_{c}^{b}\left(\lambda^{-1} \lambda^{\prime}\right)_{a}^{c} \\
& =-\frac{\zeta^{2}}{2}\left[\left(U U^{\prime}\right)^{\prime \prime}+\left\langle\mathbf{L}^{\prime \prime}\right\rangle+\frac{1}{2}\left(\lambda^{-1} \lambda^{\prime}\left\langle\mathbf{L}^{\prime}\right\rangle-\left\langle\mathbf{L}^{\prime}\right\rangle \lambda^{-1} \lambda^{\prime}\right)\right] \\
\nabla_{a} R_{\rho}^{b} & =R_{\rho}^{\rho} \Gamma_{\rho a}^{b}-R_{c}^{b} \Gamma_{\rho a}^{c}=\frac{1}{2} R_{\rho}^{\rho}\left(\lambda^{-1} \lambda^{\prime}\right)_{a}^{b}-\frac{1}{2} R_{c}^{b}\left(\lambda^{-1} \lambda^{\prime}\right)_{a}^{c}=\frac{\zeta^{2}}{4}\left[\left(-\mathbf{X} \cdot \mathbf{X}^{\prime \prime}\right)+\left\langle\mathbf{L}^{\prime}\right\rangle\right] \lambda^{-1} \lambda^{\prime} \\
\nabla_{a} R_{b}^{\rho} & =\frac{\zeta^{4}}{4} U^{2} \lambda^{\prime}\left[-\left(\mathbf{X} \cdot \mathbf{X}^{\prime \prime}\right)+\left\langle\mathbf{L}^{\prime}\right\rangle\right]
\end{aligned}
$$

Covariant derivative of metric deviation

$$
\begin{aligned}
\nabla_{b} h_{\rho}^{a} & =-\frac{\delta U}{U}\left(\lambda^{-1} \lambda^{\prime}\right)_{b}^{a}-\frac{1}{2}\left(\lambda^{-1} \delta \lambda \lambda^{-1} \lambda^{\prime}\right)_{b}^{a}, \quad \nabla_{c} h_{b}^{a}=0, \quad \nabla_{a} h_{\rho}^{\rho}=0, \\
\nabla_{a} h_{b}^{\rho} & =-\frac{\zeta^{2}}{2} U^{2}\left(\lambda^{\prime} \lambda^{-1} \delta \lambda+2 \lambda^{\prime} \frac{\delta U}{U}\right)_{a b}, \\
\nabla_{\rho} h_{b}^{a} & =\left(h_{b}^{a}\right)^{\prime}+\frac{1}{2}\left(\lambda^{-1} \lambda^{\prime} \lambda^{-1} \delta \lambda\right)_{b}^{a}-\frac{1}{2}\left(\lambda^{-1} \delta \lambda \lambda^{-1} \lambda^{\prime}\right)_{b}^{a}, \quad \nabla_{\rho} h_{\rho}^{\rho}=\left(h_{\rho}^{\rho}\right)^{\prime}, \quad \nabla_{\rho} h_{a}^{\rho}=\nabla_{\rho} h_{\rho}^{a}=0,
\end{aligned}
$$

which imply

$$
\begin{aligned}
& \nabla_{\mu} h_{\rho}^{\mu}=\left(h_{\rho}^{\rho}\right)^{\prime}-2 \frac{U^{\prime} \delta U}{U^{2}}-\frac{1}{2} \operatorname{Tr}\left(\lambda^{-1} \lambda^{\prime} \lambda^{-1} \delta \lambda\right)=-\frac{1}{U^{2}}\left[\mathbf{X}^{\prime} \cdot \delta \mathbf{X}+2 \mathbf{X} \cdot \delta \mathbf{X}^{\prime}\right], \\
& \nabla_{\mu} h_{a}^{\mu}=0, \quad 2 \nabla_{[\rho} h_{b]}^{a}=\left(\lambda^{-1} \delta \lambda\right)_{b}^{\prime a}+\frac{1}{2}\left(\lambda^{-1} \lambda^{\prime} \lambda^{-1} \delta \lambda\right)_{b}^{a}+\frac{\delta U}{U}\left(\lambda^{-1} \lambda^{\prime}\right)_{b}^{a} .
\end{aligned}
$$

Useful vector identities:

$$
\begin{aligned}
\mathbf{L}^{2} & =U^{2}\left(U^{\prime 2}-\mathbf{X}^{\prime 2}\right), \\
\mathbf{L} \cdot \mathbf{\Sigma} & =U^{2}\left(U^{\prime} \delta U-\mathbf{X}^{\prime} \cdot \delta \mathbf{X}\right)=U^{2}\left(-U \delta U^{\prime}+\mathbf{X} \cdot \delta \mathbf{X}^{\prime}\right), \\
\mathbf{L}^{\prime} \cdot \mathbf{\Sigma} & =U \delta U \mathbf{X} \cdot \mathbf{X}^{\prime \prime}-U^{2} \mathbf{X}^{\prime \prime} \cdot \delta \mathbf{X}
\end{aligned}
$$




$$
\begin{aligned}
& \mathbf{L} \cdot \boldsymbol{\Sigma}^{\prime}=U \delta U \mathbf{X}^{\prime 2}-U^{2} \mathbf{X}^{\prime} \cdot \delta \mathbf{X}^{\prime}-U U^{\prime}\left(\mathbf{X}^{\prime} \cdot \delta \mathbf{X}-\mathbf{X} \cdot \delta \mathbf{X}^{\prime}\right), \\
& \mathbf{L}^{\prime} \cdot \boldsymbol{\Sigma}^{\prime}=U \delta U \mathbf{X}^{\prime} \cdot \mathbf{X}^{\prime \prime}-U U^{\prime} \mathbf{X}^{\prime \prime} \cdot \delta \mathbf{X}+\left(\mathbf{X} \cdot \mathbf{X}^{\prime \prime}\right) \mathbf{X} \cdot \delta \mathbf{X}^{\prime}-U^{2} \mathbf{X}^{\prime \prime} \cdot \delta \mathbf{X}^{\prime} \\
& \mathbf{L} \cdot \delta \mathbf{L}=U U^{\prime}(U \delta U)^{\prime}-U \delta U \mathbf{X}^{\prime 2}-U^{2} \mathbf{X}^{\prime} \cdot \delta \mathbf{X}^{\prime}, \\
& \mathbf{L} \cdot \mathbf{L}^{\prime}=U U^{\prime} \mathbf{X} \cdot \mathbf{X}^{\prime \prime}-U^{2} \mathbf{X}^{\prime} \cdot \mathbf{X}^{\prime \prime} \\
& \mathbf{L}^{\prime} \cdot \delta \mathbf{L}=U U^{\prime} \mathbf{X}^{\prime \prime} \cdot \delta \mathbf{X}+\left(\mathbf{X} \cdot \mathbf{X}^{\prime \prime}\right) \mathbf{X} \cdot \delta \mathbf{X}^{\prime}-U \delta U \mathbf{X}^{\prime} \cdot \mathbf{X}^{\prime \prime}-U^{2} \mathbf{X}^{\prime \prime} \cdot \delta \mathbf{X}^{\prime}, \\
& \mathbf{L} \times \boldsymbol{\Sigma}=\frac{1}{2} U^{2}\left(\delta \mathbf{L}-\boldsymbol{\Sigma}^{\prime}\right)+U U^{\prime} \boldsymbol{\Sigma}-U \delta U \mathbf{L}, \\
& \mathbf{L}^{\prime} \times \mathbf{\Sigma}=U^{2} \delta \mathbf{X} \times \mathbf{X}^{\prime \prime}+\left(\mathbf{X} \cdot \mathbf{X}^{\prime \prime}\right) \boldsymbol{\Sigma}-U \delta U \mathbf{L}^{\prime}, \\
& \mathbf{L} \times \boldsymbol{\Sigma}^{\prime}=U U^{\prime} \delta \mathbf{L}-(U \delta U)^{\prime} \mathbf{L}-U^{2} \mathbf{X}^{\prime} \times \delta \mathbf{X}^{\prime}+\mathbf{X}^{\prime 2} \boldsymbol{\Sigma}, \\
& \mathbf{L}^{\prime} \times \mathbf{\Sigma}^{\prime}=-\left(\mathbf{X} \cdot \delta \mathbf{X}^{\prime}\right) \mathbf{L}^{\prime}+U^{2} \delta \mathbf{X}^{\prime} \times \mathbf{X}^{\prime \prime}+U U^{\prime} \delta \mathbf{X} \times \mathbf{X}^{\prime \prime}-U \delta U \mathbf{X}^{\prime} \times \mathbf{X}^{\prime \prime}-\left(\mathbf{X}^{\prime \prime} \cdot \delta \mathbf{X}\right) \mathbf{L} \\
& +\left(\mathbf{X} \cdot \mathbf{X}^{\prime \prime}\right) \mathbf{X} \times \delta \mathbf{X}^{\prime}+\left(\mathbf{X}^{\prime} \cdot \mathbf{X}^{\prime \prime}\right) \mathbf{\Sigma} \\
& \mathbf{L} \times \mathbf{L}^{\prime}=U U^{\prime} \mathbf{L}^{\prime}-\left(\mathbf{X} \cdot \mathbf{X}^{\prime \prime}\right) \mathbf{L}-U^{2} \mathbf{X}^{\prime} \times \mathbf{X}^{\prime \prime}, \\
& \mathbf{L} \times \delta \mathbf{L}=\left(\delta \mathbf{X} \cdot \mathbf{X}^{\prime}-\mathbf{X} \cdot \delta \mathbf{X}^{\prime}\right) \mathbf{L}+U U^{\prime} \boldsymbol{\Sigma}^{\prime}-U^{2} \mathbf{X}^{\prime} \times \delta \mathbf{X}^{\prime}-\mathbf{X}^{\prime 2} \boldsymbol{\Sigma}, \\
& \mathbf{L}^{\prime} \times \delta \mathbf{L}=-\left(\mathbf{X} \cdot \delta \mathbf{X}^{\prime}\right) \mathbf{L}^{\prime}+U^{2} \delta \mathbf{X}^{\prime} \times \mathbf{X}^{\prime \prime}-U U^{\prime} \delta \mathbf{X} \times \mathbf{X}^{\prime \prime}+U \delta U \mathbf{X}^{\prime} \times \mathbf{X}^{\prime \prime}+\left(\mathbf{X}^{\prime \prime} \cdot \delta \mathbf{X}\right) \mathbf{L} \\
& +\left(\mathbf{X} \cdot \mathbf{X}^{\prime \prime}\right) \mathbf{X} \times \delta \mathbf{X}^{\prime}-\left(\mathbf{X}^{\prime} \cdot \mathbf{X}^{\prime \prime}\right) \mathbf{\Sigma} \\
& \mathbf{L} \times \delta \mathbf{L}^{\prime}=\left(\mathbf{X}^{\prime} \cdot \delta \mathbf{X}\right) \mathbf{L}^{\prime}-U^{2} \mathbf{X}^{\prime} \times \delta \mathbf{X}^{\prime \prime}+U U^{\prime} \mathbf{X} \times \delta \mathbf{X}^{\prime \prime}-U \delta U \mathbf{X}^{\prime} \times \mathbf{X}^{\prime \prime}-\left(\mathbf{X} \cdot \delta \mathbf{X}^{\prime \prime}\right) \mathbf{L} \\
& -\left(\mathbf{X} \cdot \mathbf{X}^{\prime \prime}\right) \delta \mathbf{X} \times \mathbf{X}^{\prime}-\left(\mathbf{X}^{\prime} \cdot \mathbf{X}^{\prime \prime}\right) \boldsymbol{\Sigma} \text {. }
\end{aligned}
$$

Note that some vector quantities may be written in terms of other ones as follows

$$
\begin{aligned}
\mathbf{L} \times \boldsymbol{\Sigma}^{\prime} & =\mathbf{L} \times \delta \mathbf{L}+U U^{\prime}(\delta \mathbf{L}-\mathbf{\Sigma})+2\left(\mathbf{X}^{\prime 2} \boldsymbol{\Sigma}-\mathbf{X}^{\prime} \cdot \delta \mathbf{X} \mathbf{L}\right), \\
\mathbf{L} \cdot \boldsymbol{\Sigma}^{\prime} & =\mathbf{L} \cdot \delta \mathbf{L}+2\left(U \delta U \mathbf{X}^{\prime 2}-U U^{\prime} \mathbf{X}^{\prime} \cdot \delta \mathbf{X}\right), \\
\mathbf{L}^{\prime} \cdot \boldsymbol{\Sigma}^{\prime} & =\mathbf{L}^{\prime} \cdot \delta \mathbf{L}+2\left[\frac{U^{\prime}}{U} \mathbf{L}^{\prime} \cdot \boldsymbol{\Sigma}-\frac{\delta U}{U} \mathbf{L} \cdot \mathbf{L}^{\prime}\right], \\
\mathbf{L}^{\prime} \times \boldsymbol{\Sigma}^{\prime} & =\mathbf{L}^{\prime} \times \delta \mathbf{L}+2\left[\frac{\delta U}{U} \mathbf{L} \times \mathbf{L}^{\prime}+\frac{U^{\prime}}{U} \mathbf{L}^{\prime} \times \boldsymbol{\Sigma}+\frac{\mathbf{L}^{\prime} \cdot \boldsymbol{\Sigma}}{U^{2}} \mathbf{L}-\frac{\mathbf{L} \cdot \mathbf{L}^{\prime}}{U^{2}} \mathbf{\Sigma}\right], \\
\mathbf{L}^{\prime} \cdot(\mathbf{L} \times \boldsymbol{\Sigma}) & =0 .
\end{aligned}
$$

Conjugation by two dimensional metric $\lambda$ :

Note that for any vector $\mathbf{A}$ one obtains

$$
\lambda\langle\mathbf{A}\rangle \lambda^{-1}=\tau^{0}\left[\langle\mathbf{A}\rangle-\frac{2}{U^{2}} \mathbf{X} \cdot \mathbf{A}\langle\mathbf{X}\rangle\right] \tau^{0},
$$

which leads to

$$
\begin{aligned}
\lambda\langle\mathbf{X}\rangle \lambda^{-1} & =-\tau^{0}\langle\mathbf{X}\rangle \tau^{0}, \quad \lambda\left\langle\mathbf{X}^{\prime}\right\rangle \lambda^{-1}=\tau^{0}\left[-\frac{2 U^{\prime}}{U}\langle\mathbf{X}\rangle+\left\langle\mathbf{X}^{\prime}\right\rangle\right] \tau^{0}, \\
\lambda\langle\mathbf{L}\rangle \lambda^{-1} & =\tau^{0}\langle\mathbf{L}\rangle \tau^{0}, \quad \lambda\left\langle\mathbf{L}^{\prime}\right\rangle \lambda^{-1}=\tau^{0}\left\langle\mathbf{L}^{\prime}\right\rangle \tau^{0}, \\
\lambda\left\langle\mathbf{L}^{\prime \prime}\right\rangle \lambda^{-1} & =\tau^{0}\left[\left\langle\mathbf{X} \times \mathbf{X}^{\prime \prime \prime}-\mathbf{X}^{\prime} \times \mathbf{X}^{\prime \prime}\right\rangle+\frac{2 U^{\prime}}{U}\left\langle\mathbf{L}^{\prime}\right\rangle-\frac{2 \mathbf{X} \cdot \mathbf{X}^{\prime \prime}}{U^{2}}\langle\mathbf{L}\rangle\right] \tau^{0} \\
& =\tau^{0}\left\langle\mathbf{L}^{\prime \prime}+\frac{2}{U^{2}} \mathbf{L} \times \mathbf{L}^{\prime}\right\rangle \tau^{0}, \\
\lambda\langle\boldsymbol{\Sigma}\rangle \lambda^{-1} & =\tau^{0}\langle\mathbf{\Sigma}\rangle \tau^{0}, \\
\lambda\left\langle\boldsymbol{\Sigma}^{\prime}\right\rangle \lambda^{-1} & =\tau^{0}\left[-\frac{2}{U^{2}} \mathbf{L} \cdot \delta \mathbf{X}\langle\mathbf{X}\rangle+\left\langle\boldsymbol{\Sigma}^{\prime}\right\rangle\right] \tau^{0}=\tau^{0}\left[\frac{2 U^{\prime}}{U}\langle\mathbf{\Sigma}\rangle-\frac{2 \delta U}{U}\langle\mathbf{L}\rangle+\langle\delta \mathbf{L}\rangle\right] \tau^{0},
\end{aligned}
$$




$$
\begin{aligned}
\lambda\left\langle\boldsymbol{\Sigma}^{\prime \prime}\right\rangle \lambda^{-1}= & \tau^{0}\left[-\frac{2}{U^{2}} \mathbf{X} \cdot \boldsymbol{\Sigma}^{\prime \prime}\langle\mathbf{X}\rangle+\left\langle\boldsymbol{\Sigma}^{\prime \prime}\right\rangle\right] \tau^{0} \\
= & \tau^{0}\left[\left\langle\delta \mathbf{L}^{\prime}\right\rangle-2\left\langle\mathbf{X}^{\prime} \times \delta \mathbf{X}^{\prime}\right\rangle-2 \frac{\delta U}{U}\left\langle\mathbf{L}^{\prime}\right\rangle-\frac{4}{U^{2}} \mathbf{X} \cdot \delta \mathbf{X}^{\prime}\langle\mathbf{L}\rangle+2 \frac{\mathbf{X} \cdot \mathbf{X}^{\prime \prime}}{U^{2}}\langle\boldsymbol{\Sigma}\rangle\right. \\
& \left.\quad+4 \frac{U^{\prime}}{U}\left\langle\mathbf{X} \times \delta \mathbf{X}^{\prime}\right\rangle\right] \tau^{0}, \\
\lambda\langle\mathbf{L} \times \boldsymbol{\Sigma}\rangle \lambda^{-1}= & -\tau^{0}[\mathbf{L} \times \mathbf{\Sigma}] \tau^{0}, \\
\lambda\left\langle\mathbf{L}^{\prime} \times \mathbf{\Sigma}\right\rangle \lambda^{-1}= & -\tau^{0}\left[\mathbf{L}^{\prime} \times \mathbf{\Sigma}\right] \tau^{0},
\end{aligned}
$$

and also

$$
\begin{aligned}
\frac{1}{2} \lambda\left(\lambda^{-1} \lambda^{\prime}\left\langle\mathbf{L}^{\prime}\right\rangle-\left\langle\mathbf{L}^{\prime}\right\rangle \lambda^{-1} \lambda^{\prime}\right) \lambda^{-1} & =\tau^{0}\left[\left\langle\mathbf{X}^{\prime} \times \mathbf{X}^{\prime \prime}\right\rangle-\frac{U^{\prime}}{U}\left\langle\mathbf{L}^{\prime}\right\rangle+\frac{\mathbf{X} \cdot \mathbf{X}^{\prime \prime}}{U^{2}}\langle\mathbf{L}\rangle\right] \tau^{0} \\
& =\tau^{0}\left\langle-\frac{1}{U^{2}} \mathbf{L} \times \mathbf{L}^{\prime}\right\rangle \tau^{0}, \\
\frac{1}{2} \lambda\left(\lambda^{-1} \lambda^{\prime}\left\langle\mathbf{L}^{\prime}\right\rangle+\left\langle\mathbf{L}^{\prime}\right\rangle \lambda^{-1} \lambda^{\prime}\right) \lambda^{-1} & =\frac{U^{\prime}}{U} \mathbf{X} \cdot \mathbf{X}^{\prime \prime}-\mathbf{X}^{\prime} \cdot \mathbf{X}^{\prime \prime}+\tau^{0} \frac{U^{\prime}}{U}\left\langle\mathbf{L}^{\prime}\right\rangle \tau^{0},
\end{aligned}
$$

\section{Vector Representation of Various Terms in the ADT Potential for NMG}

In this appendix, we present various formulae for the conjugation by two dimensional metric, $\lambda$ or useful formulae to obtain the ADT potential and then present vector representation for various terms in the ADT potential of NMG.

Useful formulae for the computation of $Q_{R}^{\mu \nu}$ :

$$
\begin{aligned}
\lambda\left(\lambda^{-1} \delta \lambda\right)^{\prime} \lambda^{-1} & =\frac{1}{U^{2}}\left[\left(U \delta U^{\prime}-U^{\prime} \delta U\right) \mathbf{1}+\tau^{0}\left(-2 \frac{\delta U}{U}\langle\mathbf{L}\rangle+\langle\delta \mathbf{L}\rangle\right) \tau^{0}\right], \\
\frac{1}{2} \operatorname{Tr}\left(\lambda^{-1} \lambda^{\prime} \lambda^{-1} \delta \lambda\right) & =\frac{2 U^{\prime}}{U^{3}} \mathbf{X} \cdot \delta \mathbf{X}-\frac{1}{U^{2}} \mathbf{X}^{\prime} \cdot \delta \mathbf{X} .
\end{aligned}
$$

Useful formulae for the computation of $Q_{K}^{\mu \nu}$ : We omit the obvious matrix indices in the right hand side of equations.

$$
\begin{aligned}
\lambda_{a c} \nabla_{[\rho} h_{d]}^{c}\left(\lambda^{-1}\right)^{d b}= & \frac{1}{4 U^{2}}\left(\mathbf{X}^{\prime} \cdot \delta \mathbf{X}+2 \mathbf{X} \cdot \delta \mathbf{X}^{\prime}\right)+\frac{1}{8 U^{2}} \tau^{0}\left\langle 3 \delta \mathbf{L}+\boldsymbol{\Sigma}^{\prime}\right\rangle \tau^{0} . \\
\lambda_{a c}^{\prime} \nabla_{[\rho} h_{d]}^{c}\left(\lambda^{-1}\right)^{d b}= & \frac{U^{\prime}}{4 U^{3}}\left(\mathbf{X}^{\prime} \cdot \delta \mathbf{X}+2 \mathbf{X} \cdot \delta \mathbf{X}^{\prime}\right)+\frac{1}{8 U^{4}} \mathbf{L} \cdot(3 \delta \mathbf{L}+\boldsymbol{\Sigma}) \\
& +\tau^{0}\left\langle\frac{U^{\prime}}{8 U^{3}}\left(3 \delta \mathbf{L}+\mathbf{\Sigma}^{\prime}\right)+\frac{1}{4 U^{4}}\left(\mathbf{X}^{\prime} \cdot \delta \mathbf{X}+2 \mathbf{X} \cdot \delta \mathbf{X}^{\prime}\right) \mathbf{L}-\frac{1}{8 U^{4}} \mathbf{L} \times\left(3 \delta \mathbf{L}+\boldsymbol{\Sigma}^{\prime}\right)\right\rangle \tau^{0} . \\
\lambda^{\prime} \lambda^{-1} \delta \lambda \lambda^{-1}= & \frac{1}{U^{2}}\left(U^{\prime} \delta U-U \delta U^{\prime}+\mathbf{X} \cdot \delta \mathbf{X}^{\prime}\right)+\tau^{0}\left\langle 2 \frac{\delta U}{U^{3}} \mathbf{L}-\frac{1}{2 U^{2}} \delta \mathbf{L}+\frac{1}{2 U^{2}} \boldsymbol{\Sigma}^{\prime}\right\rangle \tau^{0} .
\end{aligned}
$$




$$
\begin{aligned}
& \lambda\left\langle\mathbf{L}^{\prime}\right\rangle \lambda^{-1} \lambda^{\prime} \lambda^{-1} \delta \lambda \lambda^{-1}=\frac{U^{\prime}}{U^{3}} \mathbf{L}^{\prime} \cdot \boldsymbol{\Sigma}+\frac{\delta U}{U^{3}} \mathbf{L} \cdot \mathbf{L}^{\prime}-\frac{1}{U^{4}} \mathbf{L}^{\prime} \cdot(\mathbf{L} \times \boldsymbol{\Sigma}) \\
& +\tau^{0}\left\langle\left(\frac{U^{\prime} \delta U}{U^{2}}+\frac{1}{U^{4}} \mathbf{L} \cdot \boldsymbol{\Sigma}\right) \mathbf{L}^{\prime}+\frac{\delta U}{U^{3}} \mathbf{L} \times \mathbf{L}^{\prime}-\frac{U^{\prime}}{U^{3}} \mathbf{L}^{\prime} \times \boldsymbol{\Sigma}\right. \\
& \left.+\frac{1}{U^{4}} \mathbf{L}^{\prime} \times(\mathbf{L} \times \mathbf{\Sigma})\right\rangle \tau^{0}, \\
& \lambda\left\langle\mathbf{L}^{\prime}\right\rangle\left[\left(\lambda^{-1} \delta \lambda\right)^{\prime}+\frac{\delta U}{U} \lambda^{-1} \lambda^{\prime}\right] \lambda^{-1}=\frac{1}{U^{2}}\left(\mathbf{L}^{\prime} \cdot \delta \mathbf{L}-\frac{\delta U}{U} \mathbf{L} \cdot \mathbf{L}^{\prime}\right) \\
& +\tau^{0}\left[\frac{\delta U^{\prime}}{U}\left\langle\mathbf{L}^{\prime}\right\rangle-\frac{1}{U^{2}}\left(\left\langle\mathbf{L}^{\prime} \times \delta \mathbf{L}\right\rangle+\frac{\delta U}{U}\left\langle\mathbf{L} \times \mathbf{L}^{\prime}\right\rangle\right)\right] \tau^{0}, \\
& 2 \lambda\left\langle\mathbf{L}^{\prime}\right\rangle \nabla h \lambda^{-1}=\frac{1}{4 U^{2}}\left(3 \mathbf{L}^{\prime} \cdot \delta \mathbf{L}+\mathbf{L}^{\prime} \cdot \mathbf{\Sigma}^{\prime}\right) \\
& +\tau^{0}\left\langle\frac{1}{2 U^{2}}\left(\mathbf{X}^{\prime} \cdot \delta \mathbf{X}+2 \mathbf{X} \cdot \delta \mathbf{X}^{\prime}\right) \mathbf{L}^{\prime}+\frac{3}{4 U^{2}} \delta \mathbf{L} \times \mathbf{L}^{\prime}-\frac{1}{4 U^{2}} \mathbf{L}^{\prime} \times \boldsymbol{\Sigma}^{\prime}\right\rangle \tau^{0}, \\
& \lambda\left(\lambda^{-1} \delta \lambda\right)^{\prime \prime} \lambda^{-1}=\left(\frac{\delta U}{U}\right)^{\prime \prime}+\tau^{0}\left[\frac{1}{U^{2}}\left\langle\delta \mathbf{L}^{\prime}\right\rangle-\frac{2}{U^{2}}\left\langle\mathbf{X}^{\prime} \times \delta \mathbf{X}^{\prime}\right\rangle+\frac{4}{U^{4}}\left(2 U^{\prime} \delta U-\mathbf{X} \cdot \delta \mathbf{X}^{\prime}\right)\langle\mathbf{L}\rangle\right. \\
& \left.-\frac{4 U^{\prime}}{U^{3}}\left\langle\delta \mathbf{X} \times \mathbf{X}^{\prime}\right\rangle-2 \frac{\delta U}{U^{3}}\left\langle\mathbf{L}^{\prime}\right\rangle+\frac{2}{U^{4}}\left(\mathbf{X} \cdot \mathbf{X}^{\prime \prime}-U U^{\prime \prime}-U^{\prime 2}\right)\langle\boldsymbol{\Sigma}\rangle\right] \tau^{0}, \\
& =\left(\frac{\delta U}{U}\right)^{\prime \prime}+\tau^{0}\left[\frac{1}{U^{2}}\left\langle\delta \mathbf{L}^{\prime}\right\rangle+\frac{2}{U^{4}}\langle\mathbf{L} \times \delta \mathbf{L}\rangle+\frac{1}{U^{2}}\left(6 \frac{U^{\prime} \delta U}{U^{2}}-2 \frac{\delta U^{\prime}}{U}\right)\langle\mathbf{L}\rangle\right. \\
& \left.-2 \frac{U^{\prime}}{U^{3}}\langle\delta \mathbf{L}\rangle-2 \frac{\delta U}{U^{3}}\left\langle\mathbf{L}^{\prime}\right\rangle\right] \tau^{0} \\
& \lambda\left(\lambda^{-1} \lambda^{\prime} \lambda^{-1} \delta \lambda\right)^{\prime} \lambda^{-1}=\left(\frac{U^{\prime} \delta U}{U^{2}}\right)^{\prime}+\frac{1}{U^{4}} \mathbf{L}^{\prime} \cdot \mathbf{\Sigma}-2 \frac{\delta U}{U^{5}} \mathbf{L}^{2}-2 \frac{U^{\prime}}{U^{5}} \mathbf{L} \cdot \mathbf{\Sigma}+\frac{1}{U^{4}} \mathbf{L} \cdot \delta \mathbf{L} \\
& +\tau^{0}\left[\frac{1}{U^{2}}\left(\frac{\delta U^{\prime}}{U}-5 \frac{U^{\prime} \delta U}{U^{2}}\right)\langle\mathbf{L}\rangle+\frac{U^{\prime}}{U^{3}}\langle\delta \mathbf{L}\rangle+\frac{\delta U}{U^{3}}\left\langle\mathbf{L}^{\prime}\right\rangle+\frac{1}{U^{2}}\left(\frac{U^{\prime}}{U}\right)^{\prime}\right. \\
& \left.+2 \frac{U^{\prime}}{U^{5}}\langle\mathbf{L} \times \mathbf{\Sigma}\rangle-\frac{1}{U^{4}}\left\langle\mathbf{L}^{\prime} \times \mathbf{\Sigma}\right\rangle-\frac{1}{U^{4}}\langle\mathbf{L} \times \delta \mathbf{L}\rangle\right] \tau^{0} . \\
& \lambda\left(\frac{\delta U}{U} \lambda^{-1} \lambda^{\prime}\right)^{\prime} \lambda^{-1}=\left(\frac{U^{\prime} \delta U}{U^{2}}\right)^{\prime}+\tau^{0}\left[\left(\frac{\delta U^{\prime}}{U^{3}}-3 \frac{U^{\prime} \delta U}{U^{4}}\right)\langle\mathbf{L}\rangle+\frac{\delta U}{U^{3}}\left\langle\mathbf{L}^{\prime}\right\rangle\right] \tau^{0} .
\end{aligned}
$$

Note that

$$
\begin{aligned}
\lambda_{a c}\left(\nabla_{[\rho} h_{d]}^{c}\right)^{\prime} \lambda^{-1 d b}= & \frac{1}{2}\left(\frac{\delta U}{U}\right)^{\prime \prime}+\frac{3}{4}\left(\frac{U^{\prime} \delta U}{U^{2}}\right)^{\prime}+\frac{1}{4 U^{4}} \mathbf{L}^{\prime} \cdot \boldsymbol{\Sigma}-\frac{\delta U}{2 U^{5}} \mathbf{L}^{2}-\frac{U^{\prime}}{2 U^{5}} \mathbf{L} \cdot \boldsymbol{\Sigma}+\frac{1}{4 U^{4}} \mathbf{L} \cdot \delta \mathbf{L} \\
+ & \tau^{0}\left[\frac{1}{2 U^{2}}\left\langle\delta \mathbf{L}^{\prime}\right\rangle-\frac{1}{4 U^{2}}\left(\frac{\delta U}{U}\right)^{\prime}\langle\mathbf{L}\rangle-\frac{3}{4} \frac{U^{\prime}}{U^{3}}\langle\delta \mathbf{L}\rangle-\frac{1}{4} \frac{\delta U}{U^{3}}\left\langle\mathbf{L}^{\prime}\right\rangle\right. \\
& \left.+\frac{1}{4 U^{2}}\left(\frac{U^{\prime}}{U}\right)^{\prime}\langle\boldsymbol{\Sigma}\rangle+\frac{U^{\prime}}{2 U^{5}}\langle\mathbf{L} \times \mathbf{\Sigma}\rangle-\frac{1}{4 U^{4}}\left\langle\mathbf{L}^{\prime} \times \boldsymbol{\Sigma}\right\rangle+\frac{3}{4 U^{4}}\langle\mathbf{L} \times \delta \mathbf{L}\rangle\right] \tau^{0},
\end{aligned}
$$


For a convenience of our calculation, let us set

$$
\lambda_{a c}\left(\nabla_{[\rho} h_{d]}^{c}\right)^{\prime} \lambda^{-1 d b}=H+\tau^{0}\langle\mathbf{H}\rangle \tau^{0},
$$

which leads to

$$
\begin{aligned}
\lambda_{a c}^{\prime}\left(\nabla_{[\rho} h_{d]}^{c}\right)^{\prime} \lambda^{-1 d b} & =\left[\tau^{0}\left(-\frac{U^{\prime}}{U}+\frac{1}{U 2}\langle\mathbf{L}\rangle\right) \tau^{0} \lambda\right]_{a c}\left(\nabla_{[\rho} h_{d]}^{c}\right)^{\prime} \lambda^{-1 d b} \\
& =\frac{U^{\prime}}{U} H+\frac{1}{U^{2}} \mathbf{L} \cdot \mathbf{H}+\tau^{0}\left[\frac{U^{\prime}}{U}\langle\mathbf{H}\rangle+\frac{1}{U^{2}} H\langle\mathbf{L}\rangle-\frac{1}{U^{2}}\langle\mathbf{L} \times \mathbf{H}\rangle\right] \tau^{0},
\end{aligned}
$$

where

$$
\begin{aligned}
& U^{4}\left[\frac{U^{\prime}}{U} \mathbf{H}+\frac{H}{U^{2}} \mathbf{L}-\frac{1}{U^{2}} \mathbf{L} \times \mathbf{H}\right] \\
= & \frac{U U^{\prime}}{2} \delta \mathbf{L}^{\prime}-\frac{3}{4}\left(U^{\prime 2}+\frac{\mathbf{L}^{2}}{U^{2}}\right) \delta \mathbf{L}-\frac{1}{4}\left(U^{\prime} \delta U+\frac{1}{U^{2}} \mathbf{L} \cdot \mathbf{\Sigma}\right) \mathbf{L}^{\prime} \\
& +\left(U^{2} H-\frac{U U^{\prime}}{4}\left(\frac{\delta U}{U}\right)^{\prime}+\frac{U^{\prime}}{2 U^{3}} \mathbf{L} \cdot \mathbf{\Sigma}+\frac{3}{4 U^{2}} \mathbf{L} \cdot \delta \mathbf{L}\right) \mathbf{L}+\left(\frac{U U^{\prime}}{4}\left(\frac{U^{\prime}}{U}\right)^{\prime}-\frac{U^{\prime}}{2 U^{3}} \mathbf{L}^{2}+\frac{\mathbf{L} \cdot \mathbf{L}^{\prime}}{4 U^{2}}\right) \mathbf{\Sigma} \\
& -\frac{1}{2} \mathbf{L} \times \delta \mathbf{L}^{\prime}+\frac{\delta U}{4 U} \mathbf{L} \times \mathbf{L}^{\prime}+\frac{3 U^{\prime}}{2 U} \mathbf{L} \times \delta \mathbf{L}-\frac{U^{\prime}}{4 U} \mathbf{L}^{\prime} \times \mathbf{\Sigma}+\frac{1}{U^{2}}\left(U^{\prime 2}-\frac{1}{4}\left(U U^{\prime}\right)^{\prime}\right) \mathbf{L} \times \mathbf{\Sigma} .
\end{aligned}
$$

Some other useful formulae to obtain $Q_{K}^{\mu \nu}$ :

$$
\begin{aligned}
& \left(\lambda^{\prime} \lambda^{-1} \lambda^{\prime}\right)_{c}^{a} \nabla_{[\rho} h_{b]}^{c} \lambda^{-1 b t}=\frac{U^{2} U^{\prime 2}+\mathbf{L}^{2}}{4 U^{6}}\left(\mathbf{X}^{\prime} \cdot \delta \mathbf{X}+2 \mathbf{X} \cdot \delta \mathbf{X}^{\prime}\right)+\frac{U^{\prime}}{4 U^{5}} \mathbf{L} \cdot\left(3 \delta \mathbf{L}+\Sigma^{\prime}\right) \\
& +\tau^{0}\left\langle\frac{U^{\prime}}{2 U^{5}}\left(\mathbf{X}^{\prime} \cdot \delta \mathbf{X}+2 \mathbf{X} \cdot \delta \mathbf{X}^{\prime}\right) \mathbf{L}+\frac{1}{8 U^{6}}\left(U^{2} U^{\prime 2}+\mathbf{L}^{2}\right)\left(3 \delta \mathbf{L}+\mathbf{\Sigma}^{\prime}\right)\right. \\
& \left.-\frac{U^{\prime}}{4 U^{5}} \mathbf{L} \times\left(3 \delta \mathbf{L}+\mathbf{\Sigma}^{\prime}\right)\right\rangle \tau^{0} \\
& \lambda_{b}^{\prime}\left[\frac{U^{\prime}}{U} \nabla_{[\rho} h_{a]}^{b}+\frac{1}{2}\left(\lambda^{-1} \lambda^{\prime}\right)_{c}^{b} \nabla_{[\rho} h_{a]}^{c}-\frac{1}{2} \nabla_{[\rho} h_{c]}^{b}\left(\lambda^{-1} \lambda^{\prime}\right)_{a}^{c}\right] \lambda^{-1 a t} \\
& =\frac{U^{\prime 2}}{4 U^{4}}\left(\mathbf{X}^{\prime} \cdot \delta \mathbf{X}+2 \mathbf{X} \cdot \delta \mathbf{X}^{\prime}\right)+\frac{U^{\prime}}{8 U^{5}} \mathbf{L} \cdot\left(3 \delta \mathbf{L}+\mathbf{\Sigma}^{\prime}\right) \\
& +\tau^{0}\left\langle\frac{1}{8 U^{4}}\left(U^{\prime 2}+\frac{\mathbf{L}^{2}}{U^{2}}\right)\left(3 \delta \mathbf{L}+\boldsymbol{\Sigma}^{\prime}\right)+\left(\frac{U^{\prime}}{4 U^{5}}\left(\mathbf{X}^{\prime} \cdot \delta \mathbf{X}+2 \mathbf{X} \cdot \delta \mathbf{X}^{\prime}\right)-\frac{1}{8 U^{6}} \mathbf{L} \cdot\left(3 \delta \mathbf{L}+\boldsymbol{\Sigma}^{\prime}\right)\right) \mathbf{L}\right. \\
& \left.-\frac{U^{\prime}}{4 U^{5}} \mathbf{L} \times\left(3 \delta \mathbf{L}+\mathbf{\Sigma}^{\prime}\right)\right\rangle \tau^{0} \\
& \frac{1}{2} \lambda^{\prime} \lambda^{-1} \lambda^{\prime}\left[\lambda^{-1} \lambda^{\prime}, \lambda^{-1} \delta \lambda\right] \lambda^{-1}=-\frac{1}{U^{4}} \tau^{0}\left\langle\frac{1}{U^{2}}\left(U^{\prime 2}+\frac{\mathbf{L}^{2}}{U^{2}}\right) \mathbf{L} \times \boldsymbol{\Sigma}+2 \frac{U^{\prime}}{U}\left(\frac{\mathbf{L} \cdot \boldsymbol{\Sigma}}{U^{2}} \mathbf{L}-\frac{\mathbf{L}^{2}}{U^{2}} \boldsymbol{\Sigma}\right)\right\rangle \tau^{0} .
\end{aligned}
$$

Now, we present various formulae to obtain vector expression for each term in the ADT potential. Since $h=0$ in this formalism, the relevant terms in the calculation of $Q_{K}^{\rho t}$ are the following eleven 
ones:

$$
\begin{aligned}
& \nabla^{2} Q_{R}^{\rho t}=\frac{\zeta^{4}}{2} k^{t}\left[-U^{2}\left(\mathbf{X} \cdot \delta \mathbf{X}^{\prime}\right)^{\prime \prime}-U U^{\prime}\left(\mathbf{X} \cdot \delta \mathbf{X}^{\prime}\right)^{\prime}+\frac{\left(U U^{\prime}\right)^{\prime}}{2} \mathbf{X} \cdot \delta \mathbf{X}^{\prime}+\mathbf{L} \cdot \delta \mathbf{L}^{\prime}+\frac{1}{2} \mathbf{L}^{\prime} \cdot \delta \mathbf{L}\right] \\
& +\frac{\zeta^{4}}{2}\left[k \tau ^ { 0 } \left\langleU^{2} \delta \mathbf{L}^{\prime \prime}+U U^{\prime} \delta \mathbf{L}^{\prime}-\frac{\left(U U^{\prime}\right)^{\prime}}{2} \delta \mathbf{L}-\left(\mathbf{X} \cdot \delta \mathbf{X}^{\prime}\right)^{\prime} \mathbf{L}\right.\right. \\
& \left.\left.-\frac{1}{2}\left(\mathbf{X} \cdot \delta \mathbf{X}^{\prime}\right) \mathbf{L}^{\prime}+\mathbf{L} \times \delta \mathbf{L}^{\prime}-\frac{1}{2} \delta \mathbf{L} \times \mathbf{L}^{\prime}\right\rangle \tau^{0}\right]^{t} \\
& -2 Q^{\alpha[\rho} R_{\alpha}^{t]}=\frac{\zeta^{4}}{4} k^{t}\left[\left(3\left(U U^{\prime}\right)^{\prime}-\mathbf{X}^{\prime 2}\right) \mathbf{X} \cdot \delta \mathbf{X}^{\prime}-\mathbf{L}^{\prime} \cdot \delta \mathbf{L}\right] \\
& +\frac{\zeta^{4}}{4}\left[k \tau^{0}\left\langle\left(\mathbf{X} \cdot \delta \mathbf{X}^{\prime}\right) \mathbf{L}^{\prime}-\left(3\left(U U^{\prime}\right)^{\prime}-\mathbf{X}^{\prime 2}\right) \delta \mathbf{L}+\delta \mathbf{L} \times \mathbf{L}^{\prime}\right\rangle \tau^{0}\right]^{t} \\
& \frac{1}{8} Q_{R^{2}}^{\rho t}=\frac{\zeta^{4}}{16} k^{t}\left[\left(4\left(U U^{\prime}\right)^{\prime}-\mathbf{X}^{\prime 2}\right)\left(\mathbf{X} \cdot \delta \mathbf{X}^{\prime}\right)+4 U^{2}\left(2(U \delta U)^{\prime \prime \prime}-\left(\mathbf{X}^{\prime} \cdot \delta \mathbf{X}^{\prime}\right)^{\prime}\right)\right. \\
& \left.-2 U U^{\prime}\left(2(U \delta U)^{\prime \prime}-\left(\mathbf{X}^{\prime} \cdot \delta \mathbf{X}^{\prime}\right)\right)+4 U \delta U\left(2\left(U U^{\prime}\right)^{\prime \prime}-\mathbf{X}^{\prime} \cdot \mathbf{X}^{\prime \prime}\right)\right] \\
& -\frac{\zeta^{4}}{16}\left[k \tau^{0}\left\langle\left(4\left(U U^{\prime}\right)^{\prime}-\mathbf{X}^{\prime 2}\right) \delta \mathbf{L}+2\left(2(U \delta U)^{\prime \prime}-\mathbf{X}^{\prime} \cdot \delta \mathbf{X}^{\prime}\right) \mathbf{L}\right\rangle \tau^{0}\right]^{t} \\
& -2 \nabla^{\alpha} \xi^{\beta} \nabla_{\alpha} \nabla^{[\rho} h_{\beta}^{t]} \\
& =-\zeta^{4} k^{t}\left[\frac{1}{2} \mathbf{L} \cdot \delta \mathbf{L}^{\prime}+\frac{U U^{\prime}}{2}(U \delta U)^{\prime \prime}-\frac{1}{8}\left(12 U^{\prime 2}-\mathbf{X}^{\prime 2}\right)(U \delta U)^{\prime}+\frac{U^{\prime}}{4 U}\left(4 U^{\prime 2}-\left(U U^{\prime}\right)^{\prime}+2 \mathbf{X}^{\prime 2}\right) U \delta U\right. \\
& \left.+\frac{1}{8}\left(8 U^{\prime 2}-\mathbf{X}^{\prime 2}\right) \mathbf{X} \cdot \delta \mathbf{X}^{\prime}+\frac{U^{\prime}}{2 U} \mathbf{L} \cdot \delta \mathbf{L}+\frac{U^{\prime}}{4 U} \mathbf{L}^{\prime} \cdot \mathbf{\Sigma}-\frac{\delta U}{4 U} \mathbf{L} \cdot \mathbf{L}^{\prime}+\left(\frac{\left(U U^{\prime}\right)^{\prime}}{4 U^{2}}-\frac{U^{\prime 2}}{U^{2}}\right) \mathbf{L} \cdot \mathbf{\Sigma}\right] \\
& -\zeta^{4}\left[k \tau ^ { 0 } \left\langle\frac{U U^{\prime}}{2} \delta \mathbf{L}^{\prime}-\frac{1}{2} \mathbf{L} \times \delta \mathbf{L}+\frac{\delta U}{4 U} \mathbf{L} \times \mathbf{L}^{\prime}-\frac{U^{\prime}}{4 U} \mathbf{L}^{\prime} \times \boldsymbol{\Sigma}+\frac{1}{16}\left(\mathbf{X}^{\prime 2}-2\left(U U^{\prime}\right)^{\prime}\right)\left(\delta \mathbf{L}-\boldsymbol{\Sigma}^{\prime}\right)\right.\right. \\
& \left.\left.-\frac{1}{4}\left(U^{\prime} \delta U+\frac{\mathbf{L} \cdot \mathbf{\Sigma}}{U^{2}}\right) \mathbf{L}^{\prime}+\frac{\mathbf{L} \cdot \mathbf{L}^{\prime}}{4 U^{2}} \boldsymbol{\Sigma}+\left(\frac{1}{2}(U \delta U)^{\prime \prime}-\frac{1}{2} \mathbf{X}^{\prime} \cdot \delta \mathbf{X}^{\prime}+\frac{\mathbf{L}^{\prime} \cdot \boldsymbol{\Sigma}}{4 U^{2}}\right) \mathbf{L}\right\rangle \tau^{0}\right]^{t} . \\
& -4 \xi^{\alpha} R_{\alpha \beta} \nabla^{[\rho} h^{t] \beta}=\zeta^{4} k^{t}\left[\frac{\left(U U^{\prime}\right)^{\prime}}{2}\left((U \delta U)^{\prime}+\mathbf{X} \cdot \delta \mathbf{X}^{\prime}\right)+\frac{1}{4} \mathbf{L}^{\prime} \cdot\left(3 \delta \mathbf{L}+\boldsymbol{\Sigma}^{\prime}\right)\right] \\
& +\zeta^{4}\left[k \tau^{0}\left\langle\frac{\left(U U^{\prime}\right)^{\prime}}{4}\left(3 \delta \mathbf{L}+\boldsymbol{\Sigma}^{\prime}\right)+\frac{1}{2}\left((U \delta U)^{\prime}+\mathbf{X} \cdot \delta \mathbf{X}^{\prime}\right) \mathbf{L}^{\prime}-\frac{1}{4} \mathbf{L}^{\prime} \times\left(3 \delta \mathbf{L}+\boldsymbol{\Sigma}^{\prime}\right)\right\rangle \tau^{0}\right]^{t}, \\
& 2 \xi^{\alpha} h^{\beta[\rho} \nabla_{\beta} R_{\alpha}^{t]}=\zeta^{4} k^{t}\left[\left(U U^{\prime}\right)^{\prime \prime} U \delta U+\frac{1}{4}\left(\mathbf{X} \cdot \mathbf{X}^{\prime \prime}\right)\left(U^{\prime} \delta U+\frac{1}{U^{2}} \mathbf{L} \cdot \boldsymbol{\Sigma}\right)\right. \\
& \left.-\frac{1}{4}\left(\frac{U^{\prime}}{U} \mathbf{L}^{\prime} \cdot \boldsymbol{\Sigma}+\frac{\delta U}{U} \mathbf{L} \cdot \mathbf{L}^{\prime}-\frac{1}{U^{2}} \mathbf{L}^{\prime} \cdot(\mathbf{L} \times \mathbf{\Sigma})\right)\right] \\
& +\zeta^{4}\left[k \tau ^ { 0 } \left\langleU \delta U \mathbf{L}^{\prime \prime}+\frac{3}{4} \frac{\delta U}{U} \mathbf{L} \times \mathbf{L}^{\prime}-\frac{1}{4}\left(U^{\prime} \delta U+\frac{\mathbf{L} \cdot \mathbf{\Sigma}}{U^{2}}\right) \mathbf{L}^{\prime}+\frac{1}{4} \frac{U^{\prime}}{U} \mathbf{L}^{\prime} \times \mathbf{\Sigma}\right.\right. \\
& \left.\left.-\frac{\mathbf{X} \cdot \mathbf{X}^{\prime \prime}}{8}\left(\delta \mathbf{L}-\boldsymbol{\Sigma}^{\prime}\right)+\frac{1}{4}\left(2 \mathbf{X} \cdot \mathbf{X}^{\prime \prime} \frac{\delta U}{U}+\frac{\mathbf{L}^{\prime} \cdot \boldsymbol{\Sigma}}{U^{2}}\right) \mathbf{L}-\frac{\mathbf{L} \cdot \mathbf{L}^{\prime}}{4 U^{2}} \boldsymbol{\Sigma}\right\rangle \tau^{0}\right]^{t}
\end{aligned}
$$




$$
\begin{aligned}
-R h_{\alpha}^{[\rho} \nabla^{t]} \xi^{\alpha} & =\frac{\zeta^{2}}{8}\left(4 \mathbf{X} \cdot \mathbf{X}^{\prime \prime}+3 \mathbf{X}^{\prime 2}\right)\left[k\left(\mathbf{X}^{\prime} \cdot \delta \mathbf{X}\right)+k \tau^{0}\left\langle\frac{1}{2}\left(\delta \mathbf{L}-\mathbf{\Sigma}^{\prime}\right)\right\rangle \tau^{0}\right]^{t}, \\
2 \xi^{[\rho} R_{\alpha}^{t]} \nabla_{\beta} h^{\alpha \beta} & =-\frac{\zeta^{4}}{2} k^{t}\left(2 \mathbf{X} \cdot \mathbf{X}^{\prime \prime}+\mathbf{X}^{\prime 2}\right)\left(\mathbf{X}^{\prime} \cdot \delta \mathbf{X}+2 \mathbf{X} \cdot \delta \mathbf{X}^{\prime}\right), \\
2 \xi_{\alpha} R^{\alpha[\rho} \nabla_{\beta} h^{t] \beta} & =-\frac{\zeta^{4}}{2}\left[k\left(\mathbf{X} \cdot \mathbf{X}^{\prime}\right)^{\prime}+k \tau^{0}\left\langle\mathbf{L}^{\prime}\right\rangle \tau^{0}\right]^{t}\left(\mathbf{X}^{\prime} \cdot \delta \mathbf{X}+2 \mathbf{X} \cdot \delta \mathbf{X}^{\prime}\right), \\
2 h^{\alpha \beta} \xi^{[\rho} \nabla_{\alpha} R_{\beta}^{t]}= & \frac{\zeta^{4}}{2} k^{t}\left[\left(5 \mathbf{X}^{\prime} \cdot \mathbf{X}^{\prime \prime}-4\left(\mathbf{X} \cdot \mathbf{X}^{\prime}\right)^{\prime \prime}\right) U \delta U-\left(\mathbf{X} \cdot \mathbf{X}^{\prime \prime}\right) \mathbf{X}^{\prime} \cdot \delta \mathbf{X}+\left(U U^{\prime}\right) \mathbf{X}^{\prime \prime} \cdot \delta \mathbf{X}\right] \\
-\left(\delta R+2 R^{\alpha \beta} h_{\alpha \beta}\right) \nabla^{[\rho} \xi^{t]}= & \frac{\zeta^{4}}{2}\left((U \delta U)^{\prime \prime}+\left(\mathbf{X} \cdot \delta \mathbf{X}^{\prime}\right)^{\prime}-\mathbf{X}^{\prime \prime} \cdot \delta \mathbf{X}\right)\left[k \mathbf{X} \cdot \mathbf{X}^{\prime}+k \tau^{0}\langle\mathbf{L}\rangle \tau^{0}\right]^{t} \cdot(\mathrm{C} .14)
\end{aligned}
$$

In the above, we have presented all the preliminary identities. All we need to do is just combining all the above results and showing the correction term in the mass formula.

\section{ADT Charges in Schwarzschild Coordinates}

As was explained in the main text, the linearized ADT formalism is insufficient to obtain the correct angular momentum of $W A d S_{3}$ black holes and even mass of static new type black holes. To obatin the correct result which is consistent with the first law of black hole thermodynamics and $A d S / C F T$ correspondence, we adopt the approach augmented by super angular momentum. However, linearized ADT formalism is enough to obtain mass and angular momentum of BTZ black holes and mass of $W A d S_{3}$ black holes. In this appendix, we obtain these charges in Schwarzschild coordinates, since these coordinates, not ones in Eq. (30), are suitable one for $A d S / C F T$ correspondence.

BTZ black holes metric may be written in the form of

$$
d s^{2}=L^{2}\left[-\frac{\left(r^{2}-r_{+}^{2}\right)\left(r^{2}-r_{-}^{2}\right)}{r^{2}} d t^{2}+\frac{r^{2}}{\left(r^{2}-r_{+}^{2}\right)\left(r^{2}-r_{-}^{2}\right)} d r^{2}+r^{2}\left(d \phi+\frac{r_{+} r_{-}}{r^{2}} d t\right)^{2}\right],
$$

where the normalization of the time translational and rotational Killing vectors is such that $\xi_{T}=$ $\frac{1}{L} \frac{\partial}{\partial t}, \xi_{R}=\frac{\partial}{\partial \phi}$. It is sufficient to take the next leading term of the metric as $h$-part for the ADT potential

$$
h_{t t}=L^{2}\left(r_{+}^{2}+r_{-}^{2}\right), \quad h_{r r}=\frac{L^{2}}{r^{4}}\left(r_{+}^{2}+r_{-}^{2}\right), \quad h_{t \phi}=L^{2} r_{+} r_{-}, \quad h_{\phi \phi}=0 .
$$

Then, the ADT potentials are given by

$$
\begin{aligned}
\left.Q_{R}^{r t}\right|_{\xi_{T}} & =\frac{1}{2 L^{3} r}\left(r_{+}^{2}+r_{-}^{2}\right), & \left.Q_{K}^{r t}\right|_{\xi_{T}} & =\frac{1}{4 L^{5} r}\left(r_{+}^{2}+r_{-}^{2}\right), \\
\left.Q_{R}^{r t}\right|_{\xi_{R}} & =\frac{r_{+} r_{-}}{L^{2} r}, & \left.Q_{K}^{r t}\right|_{\xi_{R}} & =\frac{r_{+} r_{-}}{2 L^{4} r},
\end{aligned}
$$


which gives us the mass and angular momentum of BTZ black holes in the NMG case as

$$
M=\frac{\left(r_{+}^{2}+r_{-}^{2}\right)}{8 G} \eta\left[\sigma+\frac{1}{2 m^{2} L^{2}}\right], \quad J=\frac{L r_{+} r_{-}}{4 G} \eta\left[\sigma+\frac{1}{2 m^{2} L^{2}}\right] .
$$

Note that this expression is consistent with the one obtained in various other ways [33].

The metric of $W A d S_{3}$ black holes in the Schwarzschild coordinates is given by [29]

$$
d s^{2}=L^{2}\left[-N(r)^{2} d t^{2}+R(r)^{2}\left(d \theta+N^{\theta}(r) d t\right)^{2}+\frac{d r^{2}}{4 R(r)^{2} N(r)^{2}}\right]
$$

where

$$
\begin{aligned}
N(r)^{2} & =\frac{\left(\nu^{2}+3\right)\left(r-r_{+}\right)\left(r-r_{-}\right)}{4 R(r)^{2}} \\
N^{\theta}(r) & =\frac{2 \nu r-\sqrt{r_{+} r_{-}\left(\nu^{2}+3\right)}}{2 R(r)^{2}} \\
R(r)^{2} & =\frac{r}{4}\left(3\left(\nu^{2}-1\right) r+\left(\nu^{2}+3\right)\left(r_{+}+r_{-}\right)-4 \nu \sqrt{r_{+} r_{-}\left(\nu^{2}+3\right)}\right)
\end{aligned}
$$

and $\nu^{2}=3 /\left(4 \beta^{2}-1\right)$.

It is sufficient to take the metric deviation as

$$
\begin{aligned}
h_{r r} & =\frac{1}{\nu^{2}+3} \frac{1}{r^{3}}\left(r_{+}+r_{-}\right), \\
h_{\theta \theta} & =\frac{r}{4}\left[\left(\nu^{2}+3\right)\left(r_{+}+r_{-}\right)-4 \nu \sqrt{\left(\nu^{2}+3\right) r_{+} r_{-}}\right] \\
h_{t \theta} & =-\frac{1}{2} \sqrt{\left(\nu^{2}+3\right) r_{+} r_{-}} .
\end{aligned}
$$

We can also obtain the ADT potentials as

$$
\begin{aligned}
\left.Q_{R}^{r t}\right|_{\xi_{T}} & =\frac{1}{2 L^{3}}\left(\nu^{2}+3\right)\left(r_{+}+r_{-}\right)-2 \nu \sqrt{\left(\nu^{2}+3\right) r_{+} r_{-}} \\
\left.Q_{K}^{r t}\right|_{\xi_{T}} & =\frac{1}{4 L^{5}}\left[\left(\nu^{2}+3\right)\left(4 \nu^{2}-3\right)\left(r_{+}+r_{-}\right)-4 \nu\left(16 \nu^{2}-15\right) \sqrt{\left(\nu^{2}+3\right) r_{+} r_{-}}\right] .
\end{aligned}
$$

These give us mass of $W A d S_{3}$ black holes solutions of NMG in Schwarzschild coordinates as

$$
M=\frac{L^{3}}{8 G} \eta\left[\sigma Q_{R}^{r t}+\frac{1}{m^{2}} Q_{K}^{r t}\right]=\frac{\nu\left(\nu^{2}+3\right)}{G\left(20 \nu^{2}-3\right)}\left[\nu\left(r_{+}+r_{-}\right)-\sqrt{\left(\nu^{2}+3\right) r_{+} r_{-}}\right],
$$

where we used the relation $1 / m^{2} L^{2}=2 \sigma /\left(3-20 \nu^{2}\right)$ and $\eta=\sigma=-1$ in the last equality. This result is identical with the one obtained by the $A d S / C F T$ correspondence [33]. 


\section{References}

[1] S. Deser, R. Jackiw and S. Templeton, Annals Phys. 140, 372 (1982) [Erratum-ibid. 185, 406.1988 APNYA,185,406.1988 APNYA,281,409 (1988 APNYA,185,406.1988 APNYA,2].

[2] R. Jackiw, S. Templeton and S. Deser, Phys. Rev. Lett. 48 (1982) 975.

[3] G. Clement, Class. Quant. Grav. 11 (1994) L115 arXiv:gr-qc/9404004.

[4] M. Gürses, Class. Quant. Grav. 11 (1994) 2585.

[5] K. A. Moussa, G. Clément and C. Leygnac, Class. Quant. Grav. 20 (2003) L277 arXiv:gr-qc/0303042.

[6] A. Bouchareb and G. Clément, Class. Quant. Grav. 24 (2007) 5581 arXiv:0706.0263 [gr-qc]].

[7] K. Hotta, Y. Hyakutake, T. Kubota and H. Tanida, JHEP 0807 (2008) 066 arXiv:0805.2005 [hep-th]].

[8] I. Vuorio, Phys. Lett. B 163 (1985) 91.

[9] R. Percacci, P. Sodano and I. Vuorio, Annals Phys. 176 (1987) 344.

[10] Y. Nutku, Class. Quant. Grav. 10 (1993) 2657.

[11] K. Skenderis, M. Taylor and B. van Rees, JHEP 0909 (2009) 045 [arXiv:0906.4926 [hep-th]].

[12] M. Henningson and K. Skenderis, JHEP 9807 (1998) 023 arXiv:hep-th/9806087].

[13] V. Balasubramanian and P. Kraus, Commun. Math. Phys. 208 (1999) 413 arXiv:hep-th/9902121.

[14] S. de Haro, S. N. Solodukhin and K. Skenderis, Commun. Math. Phys. 217 (2001) 595 arXiv:hep-th/0002230.

[15] E. A. Bergshoeff, O. Hohm and P. K. Townsend, Phys. Rev. Lett. 102 (2009) 201301 arXiv:0901.1766 [hep-th]].

[16] E. A. Bergshoeff, O. Hohm and P. K. Townsend, Phys. Rev. D 79 (2009) 124042 arXiv:0905.1259 [hep-th]].

[17] R. Andringa, E. Bergshoeff, M. de Roo, O. Hohm, E. Sezgin, P. Townsend, Class. Quant. Grav. 27 (2010) 025010 arXiv:0907.4658 [hep-th]].

[18] E. Bergshoeff, O. Hohm, P. Townsend, Annals Phys. 325 (2010) 1118 arXiv:0911.3061 [hepth]].

[19] E. Bergshoeff, O. Hohm and P. Townsend, arXiv:0912.2944 [hep-th]].

[20] G. Clément, Class. Quant. Grav. 26 (2009) 105015 [arXiv:0902.4634 [hep-th]].

[21] H. Ahmedov and A. N. Aliev, arXiv:1008.0303 [hep-th]].

[22] H. Ahmedov and A. N. Aliev, arXiv:1006.4264 [hep-th]]. 
[23] A. Ghodsi and M. Moghadassi, arXiv:1007.4323 [hep-th]].

[24] J. Oliva, D. Tempo and R. Troncoso, JHEP 0907 (2009) 011 [arXiv:0905.1545 [hep-th]].

[25] G. Giribet, J. Oliva, D. Tempo and R. Troncoso, Phys. Rev. D 80 (2009) 124046 arXiv:0909.2564 [hep-th]].

[26] L. Abbott and S. Deser, Phys. Lett. B 116 (1982) 259.

[27] S. Deser and B. Tekin, Phys. Rev. Lett. 89 (2002) 101101 arXiv:hep-th/0205318.

[28] S. Deser and B. Tekin, Phys. Rev. D 67 (2003) 084009 arXiv:hep-th/0212292.

[29] D. Anninos, W. Li, M. Padi, W. Song and A. Strominger, JHEP 0903 (2009) 130 arXiv:0807.3040 [hep-th]].

[30] O. Miskovic and R. Olea, JHEP 0912 (2009) 046 [arXiv:0909.2275 [hep-th]].

[31] G. Giribet and M. Leston, arXiv:1006.3349 [hep-th]].

[32] O. Hohm and E. Tonni, JHEP 1004 (2010) 093 [arXiv:1001.3598 [hep-th]].

[33] S. Nam, J. D. Park and S. H. Yi, JHEP 1007 (2010) 058 [arXiv:1005.1619 [hep-th]].

[34] S. Deser, B. Tekin, Class. Quant. Grav. 20 (2003) L259 arXiv:gr-qc/0307073.

[35] G. Clément, Phys. Rev. D 49 (1994) 5131.

[36] K. A. Moussa, G. Clement, H. Guennoune and C. Leygnac, Phys. Rev. D 78 (2008) 064065 arXiv:0807.4241 [gr-qc]]. 